\title{
ON THE THEORY OF MATRIX-VALUED FUNCTIONS BELONGING TO THE SMIRNOV CLASS
}

\author{
V.E. Katsnelson and B. Kirstein
}

A theory of matrix-valued functions from the matricial Smirnov class $\mathfrak{N}_{n}^{+}(\mathbb{D})$ is systematically developed. In particular, the maximum principle of V.I.Smirnov, inner-outer factorization, the Smirnov-Beurling characterization of outer functions and an analogue of Frostman's theorem are presented for matrix-valued functions from the Smirnov class $\mathfrak{N}_{n}^{+}(\mathbb{D})$. We also consider a family $F_{\lambda}=F-\lambda I$ of functions belonging to the matricial Smirnov class which is indexed by a complex parameter $\lambda$. We show that with the exception of a "very small" set of such $\lambda$ the corresponding inner factor in the inner-outer factorization of the function $F_{\lambda}$ is a Blaschke-Potapov product.

The main goal of this paper is to provide users of analytic matrix-function theory with a standard source for references related to the matricial Smirnov class.

NOTATIONS: $\mathbb{C}-$ the complex plane.

$\mathbb{T}:=\{t \in \mathbb{C} \quad: \quad|t|=1\} \quad$ - the unit circle.

$\mathbb{D}:=\{z \in \mathbb{C} \quad: \quad|z|<1\} \quad$ - the unit disc.

$\mathfrak{B}_{\mathbb{T}} \quad$ - the $\sigma$ - algebra of Borel subsets of $\mathbb{T}$.

$m$ - normalized Lebesgue measure on the measurable space $\left(\mathbb{T}, \mathfrak{B}_{\mathbb{T}}\right)$.

$\mathbb{C}^{n}$ - the $n$-dimensional complex space equipped with the usual Euclidean norm, i.e., for $x=\left(\xi_{1}, \ldots, \xi_{n}\right)^{\top}$ we define $\|x\|_{\mathbb{C}^{n}}:=\left\{\sum_{k=1}^{n}\left|\xi_{k}\right|^{2}\right\}^{1 / 2}$.

$\mathfrak{M}_{n} \quad$ - the set of all complex $n \times n$ matrices equipped with the standard matrix norm, namely if $M \in \mathfrak{M}_{n}$ then $\|M\|:=\sup _{x \in \mathbb{C}^{n} \backslash\{0\}}\|M x\|_{\mathbb{C}^{n}} /\|x\|_{\mathbb{C}^{n}}$.

$\mathfrak{C}_{n}:=\left\{M \in \mathfrak{M}_{n} \quad: \quad\|M\| \leq 1\right\} \quad$ - the subset of all contractive matrices in $\mathfrak{M}_{n}$. $I_{n} \quad$ - the $n \times n$ unit matrix.

As usual for $r \in \mathbb{R}$ we set $r^{+}:=\max \{r, 0\}$ and $r^{-}:=\max \{-r, 0\}$.

Hence, $r=r^{+}-r^{-}$and $|r|=r^{+}+r^{-}$. In particular, if $a \in(0, \infty)$, then

$$
\begin{aligned}
\ln ^{+} a=\max \{\ln a, 0\} & , \quad \ln ^{-} a=\max \left\{\ln \frac{1}{a}, 0\right\}, \\
\ln a=\ln ^{+} a-\ln ^{-} a & , \quad
\end{aligned}
$$

If $A \in \mathbb{C}^{p \times q}$, then the symbol $A^{\top}$ stands for the transposed matrix. 


\section{PREFACE}

In this paper, we discuss various aspects of a class of matrix-valued functions which is named after V.I. Smirnov who introduced it for the scalar case in his famous paper [Sm]. It should be mentioned that the scalar Smirnov class also appeared in early papers of Doob (see e.g. [Doo1], [Doo2] and the bibliographies in the monographs Collingwood and Lohwater $[\mathrm{CoLo}]$ and Noshiro $[\mathrm{No}]$ which contain references to many other related works of Doob). For a collection of basic facts on the Smirnov class and the intimately related function spaces named after Nevanlinna and Hardy we refer the reader to the monographs of P.L. Duren [Dur], J.B. Garnett [G], K. Hoffman [Hoff], P. Koosis [Koo], I.I. Privalov [Pri] and M. Rosenblum and J. Rovnyak [RoRo2]. These books concentrate more or less on function-theoretic properties of functions belonging to some of the mentioned classes. In the last two decades much progress has been made in clearing up topological and functional-analytic questions connected with the structure of the Smirnov class (see e.g. Yanagihara [Y1] - [Y10], Yanagihara and Kawase [YK], Yanagihara and Nakamura [YN], Stoll [St1], [St2], Roberts [Rob], Roberts and Stoll [RoSt1], [RoSt2], Mochizuki [Mo1], [Mo2], Helson [Hel2] - [Hel4], McCarthy [McC], Camera [Cam]).

A systematic study of the matricial Smirnov class was mainly promoted by the work of D.Z. Arov. In his paper [Ar1] on Darlington synthesis matricial generalization of V.I. Smirnov's important maximum principle was used in an essential way, namely with its aid a powerful criterion for proving the $J$-contractivity of a meromorphic matrix function was established. Moreover, D.Z. Arov's description of all Darlington representations of a given (pseudocontinuable) Schur function is based on the concept of denominators. A pair $\left[b_{1}, b_{2}\right]$ of inner matrix-valued functions of appropriate sizes is called a denominator of a given meromorphic matrix-valued function $f$ of bounded characteristic if $b_{1} f b_{2}$ belongs to the matricial Smirnov class.

Nehari interpolation and generalized bitangential Schur - Nevanlinna - Pick interpolation are other important problems which turned out to be closely related with the matricial Smirnov class. This is an immediate consequence of D.Z. Arov's work [Ar3] - [Ar9] (see also Nicolau [Nic1], [Nic2]). In his investigations on the corresponding inverse problem D.Z. Arov introduced particular subclasses of $J$-inner functions which are now called the classes of Arov-regular and Arov-singular $J$-inner functions. Here a $J$-inner function $V$ is called Arov-singular if $V$ and $V^{-1}$ belong to the matricial Smirnov class. Furthermore, a $J$-inner function $W$ is called left Arov-regular (resp. right Arov-regular) if it does not contain any nonconstant Arov-singular right (resp. left) divisors. D.Z. Arov (see [Ar3] [Ar7]) proved that each $J$-inner function $W$ admits (an essentially unique) factorizations

$$
W=W_{l, r} \cdot W_{l, s}=W_{r, s} \cdot W_{r, r}
$$

where the $J$-inner functions $W_{l, s}$ and $W_{r, s}$ are Arov-singular whereas the $J$-inner functions $W_{l, r}$ and $W_{r, r}$ are left Arov-regular and right Arov-regular, respectively. Furthermore, D.Z. Arov proved that a $J$-inner function is a left (resp. right) resolvent matrix of a completely indeterminate bitangential Schur - Nevanlinna - Pick interpolation problem if and only if it is left Arov-regular (resp. right Arov-regular). For several connections between left and right Arov-regularity we refer the reader to the papers [Kats1], [Kats2] where essential 
connections between left and right Blaschke - Potapov products were established. In this way the first author (see [Kats3], [Kats4]) was led to a weighted approximation problems for pseudocontinuable functions belonging to the Smirnov class. The papers [Kats1] -[Kats3] laid the basis for the study of an inverse problem for Arov-singular $J$-inner functions which was considered in [AFK7]. The papers [Ar2], [AFK1] - [AFK6] deal with several completion problems for $J$-inner functions with particular emphasis on various subclasses of $J$-inner functions (Smirnov type, inverse Smirnov type, Arov-singular type). Using the concept of Arov-singularity and Arov-regularity of $J$-inner functions and the approximation method created in [Kats3], A. J. Kheifets [Kh] answered a question of D. Sarason [Sar1] (see also [Sar2]) on exposed points in the Hardy space $H^{1}(\mathbb{D})$. Prediction theory for multivariate stationary sequences formed an important source for the development of the theory of matrix-valued holomorphic functions (see Wiener and Masani [WM1], [WM2], Helson and Lowdenslager [HL1], [HL2], Rozanov [Roz1], [Roz2], Masani [Ma1] - [Ma4]). In particular, the matricial Hardy class $H_{n}^{2}(\mathbb{D})$ (see Definition 5.1 below) became an essential tool. It turned out that the basic problems of prediction theory could be reformulated as analytic problems for appropriate functions belonging to the Hardy class $H_{n}^{2}(\mathbb{D})$. Using functional-analytic methods, Beurling's inner-outer factorization was generalized to $H_{n}^{2}(\mathbb{D})$ (see Masani [Ma2], Rozanov [Roz1]). Moreover classical results due to Szegö [Sz1] - [Sz3], Kolmogorov [Kol] and Krein [Kr] were extended to the multivariate case. Here, it turned out (see Devinatz [De]) that the matrix version of Szegö's factorization theorem and other results due to Wiener and Masani [WM1], [WM2] and Helson and Lowdenslager [HL1], [HL2] are not so much generalizations of Szegö's classical results as consequences of it. An algebraic treatment of this theory was given by Helson [Hel1]. Carrying on from the theory of matrix-valued functions belonging to the Hardy class $H_{n}^{2}(\mathbb{D})$, we will study various aspects of outer functions from the matricial Smirnov class in this paper. In particular, we will extend the theory of inner-outer factorization to the matricial Smirnov class. A central topic in our investigations is to describe the situation where the inner factor in the inner-outer factorization of a matrix-valued Smirnov class function is a Blaschke - Potapov product. Moreover, we will consider a family of functions belonging to the matricial Smirnov class which is indexed by a complex parameter $\lambda$. Then it will be shown that with exception of a "very small" set of such parameters $\lambda$ the corresponding inner factor in the inner-outer factorization of the function $F_{\lambda}$ is a Blaschke - Potapov product. Our methods to prove this use a matrix generalization of logarithmic potentials. In this way, we obtain a generalization of a classical theorem of Frostman [Fr] (see also Heins [Hei] and Rudin [Ru1], [Ru2]). It should be mentioned that it was Yu. P. Ginzburg who was a pioneer in matrix (and in operator) generalizations of Frostman's results (see [Gi6] and [GiTa1] - [GiTa3]).

\section{ON THE MATRICIAL NEVANLINNA AND SMIRNOV CLASSES}

For $F: \mathbb{D} \rightarrow \mathfrak{M}_{n}$ and $r \in[0,1)$, we define the function $F_{[r]}: \mathbb{T} \rightarrow \mathfrak{M}_{n}$ via $t \rightarrow F(r t)$.

DEFINITION 1.1. A matrix-valued function $F: \mathbb{D} \rightarrow \mathfrak{M}_{n}$ is said to belong to the matricial Nevanlinna class $\mathfrak{N}_{n}(\mathbb{D})$ if $F$ is holomorphic in $\mathbb{D}$ and if the family $\left(\ln ^{+}\left\|F_{[r]}\right\|\right)_{r \in[0,1)}$ 
is bounded in $\mathcal{L}^{1}(m)$, or more precisely, if

$$
\sup _{r \in[0,1)} \int_{\mathbb{T}} \ln ^{+}\left\|F_{[r]}(t)\right\| m(d t)<+\infty .
$$

REMARK 1.1. Let $F: \mathbb{D} \rightarrow \mathfrak{M}_{n}$ be a matrix-valued function which is holomorphic in $\mathbb{D}$. Then $F$ belongs to $\mathfrak{N}_{n}(\mathbb{D})$ if and only if the (subharmonic) function $\ln ^{+}\|F\|$ has a harmonic majorant in $\mathbb{D}$.

The definition of the Smirnov class $\mathfrak{N}^{+}(\mathbb{D})$ and of its matricial analogue $\mathfrak{N}_{n}^{+}(\mathbb{D})$ are connected with the notion of uniform integrability. Since this notion is not used very often we give the definition.

DEFINITION OF UNIFORM INTEGRABILITY. Let $(\Omega, \mathfrak{A}, \mu)$ be a measure space. Then the family $\left(f_{\alpha}\right)_{\alpha \in A}$ belonging to $\mathcal{L}^{1}(\Omega, \mathfrak{A}, \mu ; \mathbb{C})$ is called uniformly integrable with respect to $\mu$ if the following conditions are satisfied :

$$
\sup _{\alpha \in A} \int_{\Omega}\left|f_{\alpha}(t)\right| \mu(d t)<+\infty .
$$

(ii) For every $\epsilon \in(0, \infty)$ there exists a $\delta \in(0, \infty)$ (which depends only on $\epsilon$ ) such that for all $\alpha \in A$ and for all $\Delta \in \mathfrak{A}$, with $\mu(\Delta)<\delta$, the inequality

$$
\int_{\Delta}\left|f_{\alpha}(t)\right| \mu(d t)<\epsilon
$$

is fulfilled.

REMARK 1.2. If $\mu(\Omega)<+\infty$ and if for each fixed $\delta \in(0, \infty)$ there exist an $N(\delta) \in$ $\mathbb{N}$ and a sequence $\left(X_{k, \delta}\right)_{k=1}^{N(\delta)}$ from $\Delta$ such that $\Omega=\bigcup_{k=1}^{N(\delta)} \Omega_{k, \delta}$ and $\mu\left(\Omega_{k, \delta}\right) \leq \delta$ for all $k \in\{1,2, \ldots, N(\delta)\}$, then a family of functions for which condition (ii) in the preceding definition is fulfilled, automatically satisfies condition (i) . Consequently, in the case of a finite measure space $(\Omega, \mathfrak{A}, \mu)$ condition (i) can be omitted in the definition of uniform integrability. A special case of such a measure space is the Lebesgue space on $\mathbb{T}$, where $\mathfrak{A}$ is the $\sigma$-algebra of Borel subsets of $\mathbb{T}$ and $m$ is the normalized Lebesgue measure on $\mathbb{T}$.

In the sequel we will repeatedly use the following theorem from measure theory which goes back to G. Vitali [Vit] (see also [Ru3, p.133, Exercise 10]).

VITALI'S CONVERGENCE THEOREM. Let $(\Omega, \mathfrak{A}, \mu)$ be a finite measure space (i.e., $\mu(\Omega)<\infty)$. Let $\left(f_{n}\right)_{n \in \mathbb{N}}$ be a sequence from $\mathcal{L}^{1}(\Omega, \mathfrak{A}, \mu ; \mathbb{C})$ which is uniformly integrable with respect to $\mu$ and converges $\mu$-a.e. to a Borel measurable function $f: \Omega \rightarrow \mathbb{C}$.

Then $f \in \mathcal{L}^{1}(\Omega, \mathfrak{A}, \mu ; \mathbb{C})$,

$$
\lim _{n \rightarrow \infty} \int_{\Omega}\left|f_{n}-f\right| d \mu=0
$$

and

$$
\lim _{n \rightarrow \infty} \int_{\Omega} f_{n} d \mu=\int_{\Omega} f d \mu
$$


PROOF. Let $\epsilon \in(0, \infty)$. In view of the uniform $\mu$-integrability of $\left(f_{n}\right)_{n \in \mathbb{N}}$ there exists a number $\delta \in(0, \infty)$ such that for all $n \in \mathbb{N}$ and for all $\Delta \in \mathfrak{A}$, which satisfy $\mu(\Delta)<\delta$, the inequality

$$
\int_{\Delta}\left|f_{n}\right| d \mu<\frac{\epsilon}{3}
$$

is satisfied. Since $\mu(\Omega)<\infty$, Egorov's Theorem guarantees the existence of a set $B_{\delta} \in \mathfrak{A}$ such that

$$
\mu\left(B_{\delta}\right)<\delta
$$

and

$$
\lim _{n \rightarrow \infty} \sup _{w \in \Omega \backslash B_{\delta}}\left|f_{n}(\omega)-f(\omega)\right|=0 .
$$

Thus, there exists an $n_{0} \in \mathbb{N}$ such that for all $n \geq n_{0}$ and all $\omega \in \Omega \backslash B_{\delta}$ the inequality

$$
\left|f_{n}(\omega)-f(\omega)\right|<\frac{\epsilon}{3[1+\mu(\Omega)]}
$$

is satisfied. In view of (1.2) and (1.3) for $n \in \mathbb{N}$ we have

$$
\int_{B_{\delta}}\left|f_{n}\right| d \mu<\frac{\epsilon}{3} .
$$

¿From Fatou's Theorem and (1.6) we obtain

$$
\int_{B_{\delta}}|f| d \mu \leq \underline{\lim _{n \rightarrow \infty}} \int_{B_{\delta}}\left|f_{n}\right| d \mu \leq \frac{\epsilon}{3} .
$$

Combining (1.5) - (1.7) we obtain the estimate

$$
\begin{aligned}
\int_{\Omega}\left|f_{n}-f\right| d \mu & =\int_{\Omega \backslash B_{\delta}}\left|f_{n}-f\right| d \mu+\int_{B_{\delta}}\left|f_{n}-f\right| d \mu \\
& \leq \frac{\epsilon}{3[1+\mu(\Omega)]} \mu\left(\Omega \backslash B_{\delta}\right)+\int_{B_{\delta}}|f| d \mu+\int_{B_{\delta}}\left|f_{n}\right| d \mu \\
& <\frac{\epsilon}{3}+\frac{\epsilon}{3}+\frac{\epsilon}{3}=\epsilon
\end{aligned}
$$

for $n \geq n_{0}$. Thus,

$$
\lim _{n \rightarrow \infty} \int_{\Omega}\left|f_{n}-f\right| d \mu=0 .
$$

From this, all the remaining assertions follow immediately.

DEFINITION 1.2. A function $\varphi: \mathbb{R} \rightarrow \mathbb{R}$ is called strongly convex if it has the following properties: 
(i) $\varphi$ is convex.

(ii) $\varphi$ is monotonically nondecreasing.

(iii) $\varphi$ takes its values in $[0, \infty)$.

(iv) $\lim _{x \rightarrow \infty} \frac{\varphi(x)}{x}=\infty$.

(v) For some $c \in(0, \infty)$ there exist constants $M \in[0, \infty)$ and $a \in \mathbb{R}$ such that $\varphi(t+$ $c) \leq M \cdot \varphi(t)$ for all $t \in[a, \infty)$.

If (v) holds for just one value of $c \in(0, \infty)$, say $c=c_{0}$, then by (ii) it holds for all $c \in\left(0, c_{0}\right)$. By iteration it holds for $c=n c_{0}, n \in \mathbb{N}$ and hence it holds for all $c \in(0, \infty)$.

THEOREM 1.1. (de la Vallée Poussin [LVP1], Nagumo [Na].) Let $(\Omega, \mathfrak{A}, \mu)$ be a (finite or infinite) measure space, and let $\left(f_{\alpha}\right)_{\alpha \in A}$ be a family of functions belonging to $\mathcal{L}^{1}(\Omega, \mathfrak{A}, \mu ; \mathbb{C})$. In case $\mu(\Omega)=+\infty$, we assume also that

$$
\sup _{\alpha \in A} \int_{\Omega}\left|f_{\alpha}\right| d \mu<\infty .
$$

(i) Suppose that there exists a function $\varphi:[0, \infty) \rightarrow[0, \infty)$ satisfying

$$
\lim _{x \rightarrow+\infty} \frac{\varphi(x)}{x}=+\infty
$$

and

$$
\sup _{\alpha \in A} \int_{\Omega} \varphi\left(\left|f_{\alpha}\right|\right) d \mu<+\infty .
$$

Then the family $\left(f_{\alpha}\right)_{\alpha \in A}$ is uniformly integrable with respect to $\mu$.

(ii) Suppose that the family $\left(f_{\alpha}\right)_{\alpha \in A}$ is uniformly integrable with respect to $\mu$. Then there exists a strongly convex function $\varphi: \mathbb{R} \rightarrow \mathbb{R}$ such that

$$
\sup _{\alpha \in A} \int_{\Omega} \varphi\left(\left|f_{\alpha}\right|\right) d \mu<+\infty \text {. }
$$

For a modern proof of Theorem 1.1 we refer to [RoRo2, Theorem 3.10] (see also Theorem 3.1.2 in [Ru2]). This modern proof based on Vitali's Convergence Theorem.

DEFINITION 1.3. A matrix-valued function $F: \mathbb{D} \rightarrow \mathfrak{M}_{n}$ is said to belong to the matricial Smirnov class $\mathfrak{N}_{n}^{+}(\mathbb{D})$ if $F$ is holomorphic in $\mathbb{D}$ and if the family $\left(\ln ^{+}\left\|F_{[r]}\right\|\right)_{r \in[0,1)}$ is uniformly integrable with respect to the normalized Lebsgue measure $m$, i.e., if for each $\epsilon \in(0, \infty)$ there exists a $\delta \in(0, \infty)$ (which depends only on $\epsilon$ ) such that for all $r \in[0,1$ ) and for all Borel subsets $\Delta$ of $\mathbb{T}$ satisfying $m(\Delta)<\delta$ the inequality

$$
\int_{\Delta} \ln ^{+}\left\|F_{[r]}(t)\right\| m(d t)<\epsilon
$$


is fulfilled.

REMARK 1.3. In view of Remark 1.2, each matrix-valued function $F \in \mathfrak{N}_{n}^{+}(\mathbb{D})$ satisfies condition (1.1). Hence, the matricial Smirnov class $\mathfrak{N}_{n}^{+}(\mathbb{D})$ is a subclass of the matricial Nevanlinna class $\mathfrak{N}_{n}(\mathbb{D})$ :

$$
\mathfrak{N}_{n}^{+}(\mathbb{D}) \subseteq \mathfrak{N}_{n}(\mathbb{D})
$$

For a matrix-valued function $F$ belonging to $\mathfrak{N}_{n}(\mathbb{D})$ we denote by $\underline{F}: \mathbb{T} \rightarrow \mathfrak{M}_{n}$ a boundary limit function associated with $F$, i.e., $\underline{F}$ is a Borel measurable function and there exists a Borel subset $\Delta_{0}$ of $\mathbb{T}$ satisfying $m\left(\Delta_{0}\right)=0$ such that for all $t \in \mathbb{T} \backslash \Delta_{0}$ we have

$$
\lim _{r \rightarrow 1-0} F(r t)=\underline{F}(t) .
$$

Observe that in view of Vitali's theorem a function $F \in \mathfrak{N}_{n}^{+}(\mathbb{D})$ satisfies

$$
\lim _{r \rightarrow 1-0} \int_{\mathbb{T}} \ln ^{+}\|F(r t)\| m(d t)=\int_{\mathbb{T}} \ln ^{+}\|\underline{F}(t)\| m(d t) .
$$

According to Fatou's theorem,

$$
\varliminf_{r \rightarrow 1-0} \int_{\mathbb{T}} \ln ^{-}\|F(r t)\| m(d t) \geq \int_{\mathbb{T}} \ln ^{-}\|\underline{F}(t)\| m(d t) .
$$

(where equality does not hold in general). Hence,

$$
\varlimsup_{r \rightarrow 1-0} \int_{\mathbb{T}} \ln \|F(r t)\| m(d t) \leq \int_{\mathbb{T}} \ln \|\underline{F}(t)\| m(d t) .
$$

LEMMA 1.1. A matrix-valued function $F: \mathbb{D} \rightarrow \mathfrak{M}_{n}$ belongs to the matricial class $\mathfrak{N}_{n}(\mathbb{D})$ (resp. $\mathfrak{N}_{n}^{+}(\mathbb{D})$ ) if and only if each of its entries belongs to the scalar class $\mathfrak{N}(\mathbb{D})$ $\left(\operatorname{resp} . \mathfrak{N}^{+}(\mathbb{D})\right)$.

As the determinant of a matrix is a polynomial of its elements and because each of the classes $\mathfrak{N}(\mathbb{D})$ and $\mathfrak{N}^{+}(\mathbb{D})$ is an algebra over $\mathbb{C}$ the following result holds true.

LEMMA 1.2. (i) If $F \in \mathfrak{N}_{n}(\mathbb{D})$, then $\operatorname{det} F \in \mathfrak{N}(\mathbb{D})$.

(ii) If $F \in \mathfrak{N}_{n}^{+}(\mathbb{D})$, then $\operatorname{det} F \in \mathfrak{N}^{+}(\mathbb{D})$.

As a special case of (1.10), (1.11) and (1.12) (corresponding to the scalar case) we obtain for a function $F \in \mathfrak{N}_{n}^{+}(\mathbb{D})$ from part (ii) of Lemma 1.2 that

$$
\begin{aligned}
& \lim _{r \rightarrow 1-0} \int_{\mathbb{T}} \ln ^{+}|\operatorname{det}[F(r t)]| m(d t)=\int_{\mathbb{T}} \ln ^{+}|\operatorname{det}[\underline{F}(t)]| m(d t), \\
& \underset{r \rightarrow 1-0}{\lim _{\mathbb{T}}} \int_{\mathbb{T}} \ln ^{-}|\operatorname{det}[F(r t)]| m(d t) \geq \int_{\mathbb{T}} \ln ^{-}|\operatorname{det}[\underline{F}(t)]| m(d t),
\end{aligned}
$$


and, finally, that

$$
\varlimsup_{r \rightarrow 1-0} \int_{\mathbb{T}} \ln |\operatorname{det}[F(r t)]| \quad m(d t) \leq \int_{\mathbb{T}} \ln |\operatorname{det}[\underline{F}(t)]| m(d t) .
$$

In the following we will use the Poisson kernel $P: \mathbb{D} \times \mathbb{T} \rightarrow(0, \infty)$ which is defined by the formula

$$
P(z, t):=\frac{1-|z|^{2}}{|t-z|^{2}} .
$$

THEOREM 1.2. Let $F \in \mathfrak{N}_{n}(\mathbb{D})$ with $F \not \equiv 0$ and let $u_{F}$ denote the least harmonic majorant of $\log \|F\|$. Then the following statements are equivalent:

(i) $\quad F \in \mathfrak{N}_{n}^{+}(\mathbb{D})$.

(ii) $u_{F}(z) \leq \int_{\mathbb{T}} \ln \|\underline{F}(t)\| \frac{1-|z|^{2}}{|t-z|^{2}} m(d t)$ for every $z \in \mathbb{D}$.

(iii) $\ln \|F(z)\| \leq \int_{\mathbb{T}} \ln \|\underline{F}(t)\| \frac{1-|z|^{2}}{|t-z|^{2}} m(d t) \quad$ for every $z \in \mathbb{D}$.

(iv) There exist a strongly convex function $\varphi: \mathbb{R} \rightarrow \mathbb{R}$ and a number $r_{0} \in(0,1)$ such

$$
\text { that } \sup _{r \in\left[r_{0}, 1\right)} \int_{\mathbb{T}} \varphi\left(\ln \left\|F_{[r]}(t)\right\|\right) m(d t)<+\infty .
$$

(v) There exists a strongly convex function $\psi: \mathbb{R} \rightarrow \mathbb{R}$ such that

$$
\sup _{r \in[0,1)} \int_{\mathbb{T}} \psi\left(\ln ^{+}\left\|F_{[r]}(t)\right\|\right) m(d t)<+\infty .
$$

PROOF. Theorem 1.2 can be proved by a slight modification of the proof of Theorem 3.3.5 in [Ru2]. Here, Theorem 1.1 plays an essential role.

For further results on matrix-valued functions belonging to one of the classes named after Nevanlinna, Smirnov and Hardy we refer the reader to chapter 4 in [RoRo1].

\section{MATRIX FUNCTIONS OF THE SMIRNOV CLASS AS MULTIPLES OF CONTRACTIVE MATRIX FUNCTIONS}

Recall that a scalar function $e: \mathbb{D} \rightarrow \mathbb{C}$ is said to be outer (in the sense of V.I. Smirnov) if there exist a unimodular constant $C \in \mathbb{T}$ and a function $w: \mathbb{T} \rightarrow[0, \infty)$ for which $\log w$ is $m$-integrable such that for $z \in \mathbb{D}$ the relation

$$
e(z)=C \cdot \exp \left\{\int_{\mathbb{T}} \frac{t+z}{t-z} \ln [w(t)] m(d t)\right\}
$$


holds true. Let $\mathfrak{E}(\mathbb{D})$ denote the class of all outer functions. ¿From its definition it is obvious, that the class $\mathfrak{E}(\mathbb{D})$ is multiplicative: If $e_{1}, e_{2} \in \mathfrak{E}(\mathbb{D})$, then $e_{1} \cdot e_{2} \in \mathfrak{E}(\mathbb{D})$.

The following statement is well-known (see e.g. Theorem 4.29 in [RoRo2]).

LEMMA 2.1. Let $e: \mathbb{D} \rightarrow \mathbb{C}$ be some function. Then the following statements are equivalent:

$$
\begin{aligned}
& \text { (i) } e \in \mathfrak{E}(\mathbb{D}) . \\
& \text { (ii) } e \in \mathfrak{N}^{+}(\mathbb{D}), e \not \equiv 0 \text { and } e^{-1} \in \mathfrak{N}^{+}(\mathbb{D}) .
\end{aligned}
$$

In particular, a function $e$ of type (2.1) belongs to the class $\mathfrak{N}(\mathbb{D})$ and, consequently, it possesses a boundary function $\underline{e}: \mathbb{T} \rightarrow \mathbb{C}$. It is known that for almost all $t \in \mathbb{T}$ with respect to $m$,

$$
|\underline{e}(t)|=w(t) .
$$

As the function $\ln |e|$ is harmonic in $\mathbb{D}$ we obtain

$$
\int_{\mathbb{T}} \ln |e(r t)| m(d t)=\ln |e(0)|=\int_{\mathbb{T}} \ln [w(t)] m(d t) .
$$

for $r \in[0,1)$. Consequently, if $e \in \mathfrak{E}(\mathbb{D})$, then for $r \in[0,1)$ we obtain

$$
\int_{\mathbb{T}} \ln |e(r t)| m(d t)=\int_{\mathbb{T}} \ln |\underline{e}(t)| m(d t) .
$$

Let us recall the following useful characterization of outer functions (see e.g. Corollaries 4.16 and 4.17 in [RoRo2]).

LEMMA 2.2. Let $e \in \mathfrak{N}(\mathbb{D})$ but $e \not \equiv 0$. Then the following statements are equivalent:

(i) $e$ is outer.

(ii) For all $z \in \mathbb{D}, \quad \ln |e(z)|=\int_{\mathbb{T}} \operatorname{Re} \frac{t+z}{t-z} \ln |\underline{e}(t)| m(d t)$.

(iii) There is a $z_{0} \in \mathbb{D}$ such that $\ln \left|e\left(z_{0}\right)\right|=\int_{\mathbb{T}} \operatorname{Re} \frac{t+z_{0}}{t-z_{0}} \ln |\underline{e}(t)| m(d t)$.

(iv) If $h \in \mathcal{N}^{+}(\mathbb{D})$ satisfies $|\underline{h}(t)| \leq|\underline{e}(t)|$ for almost all $t \in \mathbb{T}$ with respect to $m$, then for all $z \in \mathbb{D}$ the inequality $|h(z)| \leq|e(z)|$ holds true.

(v) If $z_{0} \in \mathbb{D}$ and if $h \in \mathcal{N}^{+}(\mathbb{D})$ satisfies $|\underline{h}(t)| \leq|\underline{e}(t)|$ for almost all $t \in \mathbb{T}$ with respect to $m$, then the inequality $\left|h\left(z_{0}\right)\right| \leq\left|e\left(z_{0}\right)\right|$ holds true.

Observe that conditions (iii) and (v) of Lemma 2.2 are usually used with the choice $z_{0}=0$. 
In the proof of Lemma 2.4 and also in further considerations we will use the following result which goes back to V.I. Smirnov [Sm].

THE MAXIMUM PRINCIPLE OF V.I. SMIRNOV. Let $f \in \mathfrak{N}^{+}(\mathbb{D})$ be such that its boundary function $\underline{f}$ is $m$-essentially bounded. Then $f$ is bounded in the unit disc and satisfies

$$
\sup _{z \in \mathbb{D}}|f(z)|=\operatorname{ess}_{t \in \mathbb{T}}|\underline{f}(t)|
$$

This result can be generalized to the matrix case.

THE MAXIMUM PRINCIPLE OF V.I. SMIRNOV FOR MATRIX FUNCTIONS. Let $F \in \mathfrak{N}_{n}^{+}(\mathbb{D})$ be such that its boundary function $\underline{F}$ satisfies $\operatorname{ess~sup}_{t \in \mathbb{T}}\|\underline{F}(t)\|<\infty$.

Then $F$ is bounded in the unit disc and satisfies

$$
\sup _{z \in \mathbb{D}}\|F(z)\|=\operatorname{ess~sup}_{t \in \mathbb{T}}\|\underline{F}(t)\| .
$$

PROOF. Let $F=\left(F_{j, k}\right)_{j, k=1}^{n}$, and fix the indices $j, k \in\{1, \ldots, n\}$. In view of the inequality $\left|F_{j, k}(z)\right| \leq\|F(z)\|(z \in \mathbb{D})$, then $F_{j, k} \in \mathfrak{N}^{+}(\mathbb{D})$ and

$$
\underset{t \in \mathbb{T}}{\operatorname{ess} \sup }\left|\underline{F_{j, k}}(t)\right| \leq \underset{t \in \mathbb{T}}{\operatorname{ess} \sup }\|\underline{F}(t)\| .
$$

According to the maximum principle for scalar functions we then have

$$
\sup _{z \in \mathbb{D}}\left|F_{j, k}(z)\right|<+\infty .
$$

Hence,

$$
\sup _{z \in \mathbb{D}}\|F(z)\|<+\infty .
$$

The bounded holomorphic matrix-valued function $F$ admits the Poisson integral representation

$$
F(z)=\int_{\mathbb{T}} \underline{F}(t) \cdot P(z, t) m(d t) \quad, \quad z \in \mathbb{D} .
$$

Therefore, by the integral version of the triangle inequality, we obtain

$$
\|F(z)\| \leq \int_{\mathbb{T}}\|\underline{F}(t)\| \cdot P(z, t) m(d t) \quad, \quad z \in \mathbb{D} .
$$

But this in turn implies the inequality $\|F(z)\| \leq \underset{t \in \mathbb{T}}{\operatorname{ess} \sup }\|\underline{F}(t)\| \quad(z \in \mathbb{D})$.

Since the function $\ln ^{+}\|F\|$ is subharmonic for an analytic matrix-valued function $F$ the following result is true.

LEMMA 2.3. Let $F \in \mathfrak{N}_{n}^{+}(\mathbb{D})$. Then for all $z \in \mathbb{D}$ the inequality

$$
\|F(z)\| \leq \exp \left\{\int_{\mathbb{T}} P(z, t) \ln \|\underline{F}(t)\| m(d t)\right\}
$$


holds true.

For a proof of Lemma 2.3 we refer to Theorem 3.13 in [RoRo2].

Clearly, the maximum principle of V.I. Smirnov is a consequence of inequality (2.4).

DEFINITION 2.1. The set $\mathfrak{S}_{n \times n}(\mathbb{D})$ of all holomorphic matrix-valued functions $S$ : $\mathbb{D} \rightarrow \mathfrak{C}_{n}$ is called the $n \times n$ Schur class.

LEMMA 2.4. A matrix-valued function $F: \mathbb{D} \rightarrow \mathfrak{M}_{n}$ belongs to the Smirnov class $\mathfrak{N}_{n}^{+}(\mathbb{D})$ if and only if it admits a representation of the form

$$
F=\frac{1}{d} \cdot \Phi,
$$

where $\Phi \in \mathfrak{S}_{n \times n}(\mathbb{D})$ and $d$ is an outer function which belongs to $\mathfrak{S}(\mathbb{D})$.

PROOF. I. Suppose that $F$ admits a representation of the form (2.5). Then $\Phi \in$ $\mathfrak{N}_{n}^{+}(\mathbb{D})$ and, as $d$ is outer, we have $d^{-1} \in \mathfrak{N}^{+}(\mathbb{D})$. Thus, as $\mathfrak{N}^{+}(\mathbb{D})$ is an algebra over $\mathbb{C}$, we get $\Phi \cdot d^{-1} \in \mathfrak{N}_{n}^{+}(\mathbb{D})$.

II. Suppose that $F \in \mathfrak{N}_{n}^{+}(\mathbb{D})$. We can assume that $F$ is not the null function in $\mathbb{D}$. Then $\ln \|\underline{F}\|$ is $m$-integrable. We define $d: \mathbb{D} \rightarrow \mathbb{C}$ via

$$
d(z):=\exp \left\{-\int_{\mathbb{T}} \frac{t+z}{t-z} \ln \|\underline{F}\| m(d t)\right\} .
$$

Then, from our earlier considerations (see (2.1) - (2.4)), it is clear that $d$ is a scalar outer function and that the corresponding boundary function $\underline{d}$ satisfies

$$
|\underline{d}(t)|=\|\underline{F}(t)\|^{-1}
$$

for almost all $t \in \mathbb{T}$ with respect to $m$. Now define $\Phi: \mathbb{D} \rightarrow \mathfrak{M}_{n}$ via

$$
\Phi(z):=d(z) \cdot F(z) .
$$

Then, since $F \in \mathfrak{N}_{n}^{+}(\mathbb{D}), d \in \mathfrak{N}^{+}(\mathbb{D})$ and $\mathfrak{N}^{+}(\mathbb{D})$ is an algebra over $\mathbb{C}$, we see that

$$
\Phi \in \mathfrak{N}_{n}^{+}(\mathbb{D}) .
$$

¿From (2.6) and (2.7) we get

$$
\|\underline{\Phi}(t)\|=1
$$

for almost all $t \in \mathbb{T}$ with respect to $m$. Finally, in view of (2.8) and (2.9), the maximum principle of V.I. Smirnov implies that for $z \in \mathbb{D}$ we obtain $\|\Phi(z)\| \leq 1$. Thus, $\Phi \in \mathfrak{S}_{n \times n}(\mathbb{D})$.

\section{OUTER MATRIX-VALUED FUNCTIONS}

The main goal of this section is to discuss outer matrix-valued functions which belong to the Smirnov class $\mathfrak{N}_{n}^{+}(\mathbb{D})$. The needs of prediction theory of multivariate stationary 
stochastic processes initiated an intensive study of matrix-valued outer functions belonging to the Hardy class $H_{n}^{2}(\mathbb{D})$ (see Definition 5.1 below) which is a subclass of $\mathfrak{N}_{n}^{+}(\mathbb{D})$. The formula for the best predictor of a multivariate stationary stochastic process of a given time in terms of its past depends in an essential manner on a particular outer matrix-valued function belonging to $H_{n}^{2}(\mathbb{D})$ (see Wiener and Masani [WM1], [WM2], Helson and Lowdenslager [HL1], [HL2], Rozanov [Roz1], [Roz2], Masani [Ma1] - [Ma4] and for operator-valued generalizations also Devinatz [De], Helson [Hel1], Sz.-Nagy and Foias [SZNF], Nikolskii [Nik2]).

DEFINITION 3.1. A matrix-valued function $E: \mathbb{D} \rightarrow \mathfrak{M}_{n}$ is called outer (in the sense of V.I. Smirnov) if $E \in \mathfrak{N}_{n}^{+}(\mathbb{D})$ and $\operatorname{det} E$ is outer. The class of all $n \times n$ matrix-valued outer functions will be denoted by $\mathfrak{E}_{n}(\mathbb{D})$.

If $E \in \mathfrak{E}_{n}(\mathbb{D})$ then, in particular, for all $z \in \mathbb{D}$ we have

$$
\operatorname{det}[E(z)] \neq 0
$$

Definition 3.1 is clearly an immediate generalization of the notion of a scalar outer function. This definition of an outer matrix-valued function enables us to avoid the study of the question of a matricial analogue of formula (2.1).

REMARK 3.1. The class $\mathfrak{E}_{n}(\mathbb{D})$ is multiplicative: If $E_{1}, E_{2} \in \mathfrak{E}_{n}(\mathbb{D})$ then $E_{1} \cdot E_{2} \in \mathfrak{E}_{n}()$.

REMARK 3.2. Let $E \in \mathfrak{E}_{n}(\mathbb{D})$. Then $E^{\top} \in \mathfrak{E}_{n}(\mathbb{D})$.

THEOREM 3.1. (Determinant characterization of outer matrix-valued functions)

(i) Let $E \in \mathfrak{E}_{n}(\mathbb{D})$. Then $\operatorname{det}[E(z)] \neq 0$ for all $z \in \mathbb{D}$ and $E^{-1} \in \mathfrak{N}_{n}^{+}(\mathbb{D})$.

(ii) Let $E$ be a function from $\mathfrak{N}_{n}^{+}(\mathbb{D})$ for which det $E$ never vanishes in $\mathbb{D}$ and $E^{-1}$ belongs to $\mathfrak{N}_{n}^{+}(\mathbb{D})$. Then $E \in \mathfrak{E}_{n}(\mathbb{D})$.

PROOF. (i) According to the rule for computing the inverse matrix we have the representation

$$
E^{-1}=\frac{1}{\operatorname{det} E} \cdot A
$$

where $A: \mathbb{D} \rightarrow \mathfrak{M}_{n}$ is a matrix-valued function the entries of which are polynomials of the elements of matrix $E$ (namely, the cofactors of the corresponding elements). Since the class $\mathfrak{N}^{+}(\mathbb{D})$ is an algebra over $\mathbb{C}$, each entry of $A$ belongs to $\mathfrak{N}^{+}(\mathbb{D})$. Hence, $A \in \mathfrak{N}_{n}^{+}(\mathbb{D})$. From the fact that $E \in \mathfrak{E}_{n}(\mathbb{D})$ and Lemma 2.1 it then follows that $(\operatorname{det} E)^{-1} \in \mathfrak{N}^{+}(\mathbb{D})$, and thus in view of $(3.1), E^{-1} \in \mathfrak{N}_{n}^{+}(\mathbb{D})$. Hence, (i) is proved.

(ii) By Lemma 1.2, det $E \in \mathfrak{N}^{+}(\mathbb{D})$ and $\operatorname{det}\left(E^{-1}\right) \in \mathfrak{N}^{+}(\mathbb{D})$. Therefore, the function $\operatorname{det} E$ satisfies condition (ii) in Lemma 2.1. Thus, $\operatorname{det} E \in \mathfrak{E}(\mathbb{D})$, and so, in view of Definition 3.1, $E \in \mathfrak{E}_{n}(\mathbb{D})$. Hence (ii) is proved.

The following result supplements the statement of Lemma 2.4.

LEMMA 3.1. Let $E \in \mathfrak{E}_{n}(\mathbb{D})$. Then $E$ has a representation of the form

$$
E=\frac{1}{d} \cdot C
$$

where $C \in \mathfrak{S}_{n \times n}(\mathbb{D}) \cap \mathfrak{E}_{n}(\mathbb{D})$ and $d \in \mathfrak{E}(\mathbb{D})$. 
PROOF. In view of Lemma 2.4, the function $E$ has a representation of the form

$$
E=\frac{1}{d} \cdot C,
$$

where $C \in \mathfrak{S}_{n \times n}(\mathbb{D})$ and $d \in \mathfrak{E}(\mathbb{D})$. Lemma 2.1 guarantees that $d^{-1} \in \mathfrak{N}^{+}(\mathbb{D})$. Since $E \in$ $\mathfrak{E}_{n}(\mathbb{D})$, it follows from Theorem 3.1 that $E^{-1} \in \mathfrak{N}_{n}^{+}(\mathbb{D})$. Therefore, as $\mathfrak{N}^{+}(\mathbb{D})$ is an algebra over $\mathbb{C}$, from $C^{-1}=d^{-1} \cdot E^{-1}$ we see that $C^{-1} \in \mathfrak{N}_{n}^{+}(\mathbb{D})$. Thus, as $C \in \mathfrak{S}_{n \times n}(\mathbb{D}) \subseteq \mathfrak{N}_{n}^{+}(\mathbb{D})$ it follows from Theorem 3.1 that $C \in \mathfrak{E}_{n}(\mathbb{D})$.

Let us recall the following notion.

DEFINITION 3.2. The class $H_{n}^{\infty}(\mathbb{D})$ consists of all matrix-valued functions $F: \mathbb{D} \rightarrow$ $\mathfrak{M}_{n}$ which are holomorphic and bounded in $\mathbb{D}$, i.e.,

$$
\sup _{z \in \mathbb{D}}\|F(z)\|<\infty .
$$

THEOREM 3.2. (i) Let $E \in \mathfrak{E}_{n}(\mathbb{D})$. Then there exists a sequence $\left(F_{k}\right)_{k \in \mathbb{N}}$ from $H_{n}^{\infty}(\mathbb{D})$ with the following properties:

$(\alpha)$ For almost all $t \in \mathbb{T}$ with respect to $m, \quad \lim _{k \rightarrow \infty} \underline{E}(t) \cdot \underline{F_{k}}(t)=I_{n}$.

( $\beta)$ The family $\left(\ln ^{+}\left\|\underline{F_{k}}\right\|\right)_{k \in \mathbb{N}}$ is uniformly integrable with respect to $\mathrm{m}$.

$(\gamma)$ There exists a Borel subset $B_{0}$ of $\mathbb{T}$ with $m\left(B_{0}\right)=0$ such that for all $k \in \mathbb{N}$ and all $t \in \mathbb{T} \backslash B_{0}$ the inequality $\left\|\underline{E}(t) \cdot \underline{F_{k}}(t)\right\| \leq 1$ holds true.

(ii) Let $E \in \mathfrak{N}_{n}^{+}(\mathbb{D})$ be such that there exists a sequence $\left(F_{k}\right)_{k \in \mathbb{N}}$ belonging to $H_{n}^{\infty}(\mathbb{D})$ satisfying the above conditions $(\alpha)$ and $(\beta)$. Then $E \in \mathfrak{E}_{n}(\mathbb{D})$.

REMARK 3.3. Theorem 3.2 expresses in some sense a Smirnov class generalization of that characterization of the property that a function is outer which is formulated in terms of the shift-invariant subspace generated by this function. Sometimes the approximation property contained in Theorem 3.2 is called weak invertibility of the function $E$ (see [Sh] or [Nik1,Ch.2]). For the spaces $H_{n}^{\infty}(\mathbb{D})$ or $H_{n}^{2}(\mathbb{D})$ this approximation property (weak invertibility) will be often used for defining the notion "outer function". Observe that in the scalar case $(n=1)$ it was already shown by V.I. Smirnov $[\mathrm{Sm}]$ that for an outer function $e$ the linear subspace $e \cdot H^{2}(\mathbb{D})$ is dense in $H^{2}(\mathbb{D})$. Concerning several generalizations of this result of V.I. Smirnov we refer the reader to chapter 2 in [Nik1] (in particular, see Theorem 3 in Section 2.2. ).

PROOF OF THEOREM 3.2. (i) Since $E$ is a matrix-valued outer function, Theorem 3.1 guarantees that $E^{-1} \in \mathfrak{N}_{n}^{+}(\mathbb{D})$. We fix a boundary function $\underline{E}$ of $E$ such $\operatorname{det}[\underline{E}(t)] \neq 0$ for $t \in \mathbb{T}$. Then for $k \in \mathbb{N}$ we define $w_{k}: \mathbb{T} \rightarrow(0, \infty)$ via

$$
w_{k}(t):=\left\{\begin{array}{cll}
1 & , \text { if } \quad\left\|\underline{E}^{-1}(t)\right\|<k \\
\frac{1}{\left\|\underline{E}^{-1}(t)\right\|} & , \text { if } \quad\left\|\underline{E}^{-1}(t)\right\| \geq k .
\end{array}\right.
$$

Clearly

$$
0<w_{1}(t) \leq w_{2}(t) \leq w_{3}(t) \leq \ldots \leq 1
$$


for $t \in \mathbb{T}$ and

$$
\lim _{k \rightarrow \infty} w_{k}(t)=1
$$

From (3.5) we see that the inequality

$$
w_{1}(t) \geq\left\|\underline{E}^{-1}(t)\right\|^{-1}
$$

holds for $t \in \mathbb{T}$. Since $E^{-1} \in \mathfrak{N}_{n}^{+}(\mathbb{D})$, we infer that

$$
\ln \left[\left\|\underline{E}^{-1}\right\|^{-1}\right] \in \mathcal{L}^{1}\left(\mathbb{T}, \mathfrak{B}_{\mathbb{T}}, m ; \mathbb{C}\right) .
$$

From (3.5) - (3.7) we obtain

$$
\int_{\mathbb{T}} \ln \left[w_{k}(t)\right] m(d t)>-\infty .
$$

Hence, for $k \in \mathbb{N}$ the function $\varphi_{k}: \mathbb{D} \rightarrow \mathbb{C}$ which is given by

$$
\varphi_{k}(z):=\exp \left\{\int_{\mathbb{T}} \frac{t+z}{t-z} \ln \left[w_{k}(t)\right] m(d t)\right\}
$$

is well-defined. Moreover from its definition it is clear that $\varphi_{k} \in \mathfrak{N}^{+}(\mathbb{D})$ (or more precisely, that $\varphi_{k}$ is even outer). In view of (3.5) and (3.6) the monotone convergence theorem guarantees that

$$
\lim _{k \rightarrow \infty} \varphi_{k}(z)=1, \quad z \in \mathbb{D} .
$$

Since $\left|\underline{\varphi_{k}}(t)\right|=w_{k}(t)$ for almost all $t \in \mathbb{T}$ with respect to $m$, formula (3.6) yields

$$
\lim _{k \rightarrow \infty}\left|\underline{\varphi_{k}}(t)\right|=1
$$

for almost all $t \in \mathbb{T}$ with respect to $m$. In view of (3.5) and (3.6), another application of the monotone convergence theorem gives us

$$
\lim _{k \rightarrow \infty} \int_{\mathbb{T}}\left|\underline{\varphi_{k}}(t)\right|^{2} m(d t)=\lim _{k \rightarrow \infty} \int_{\mathbb{T}}\left[w_{k}(t)\right]^{2} m(d t)=\int_{\mathbb{T}} 1 d m=1 .
$$

For $k \in \mathbb{N}$, we have

$$
\int_{\mathbb{T}}\left|\underline{\varphi_{k}}(t)-1\right|^{2} m(d t)=\int_{\mathbb{T}}\left|\underline{\varphi_{k}}(t)\right|^{2} m(d t)-2 \Re\left[\varphi_{k}(0)\right]+1 .
$$

Combining (3.10) - (3.12) it follows that

$$
\lim _{k \rightarrow \infty} \int_{\mathbb{T}}\left|\underline{\varphi_{k}}(t)-1\right|^{2} m(d t)=0 .
$$

In view of (3.13), the F. Riesz - Fischer theorem yields a subsequence $\left(\underline{\varphi_{l_{k}}}\right)_{k \in \mathbb{N}}$ of $\left(\underline{\varphi_{k}}\right)_{k \in \mathbb{N}}$ such that

$$
\lim _{k \rightarrow \infty} \underline{\varphi_{l_{k}}}(t)=1
$$


for almost all $t \in \mathbb{T}$ with respect to $m$. Let $k \in \mathbb{N}$ and set

$$
F_{k}:=E^{-1} \cdot \varphi_{l_{k}} .
$$

Then, since $E^{-1} \in \mathfrak{N}_{n}^{+}(\mathbb{D})$ and $\varphi_{l_{k}} \in \mathfrak{N}^{+}(\mathbb{D})$, we get $F_{k} \in \mathfrak{N}_{n}^{+}(\mathbb{D})$. Thus as $\left|\underline{\varphi_{l_{k}}}\right|=w_{l_{k}}$ almost everywhere with respect to $m$ it follows from (3.15) and (3.4) that

$$
\left\|\underline{F_{k}}(t)\right\|=w_{l_{k}}(t) \cdot\left\|\underline{E}^{-1}(t)\right\| \leq l_{k}
$$

for almost all $t \in \mathbb{T}$ with respect to $m$. Thus, the maximum principle of V.I. Smirnov implies that $\left\|F_{k}(z)\right\| \leq l_{k}$ for all $z \in \mathbb{D}$. Consequently, $F_{k} \in H_{n}^{\infty}(\mathbb{D})$. From (3.15) it follows that

$$
E \cdot F_{k}=\varphi_{l_{k}} \cdot I_{n}
$$

From (3.5) we obtain

$$
\left|\underline{\varphi_{l_{k}}}(t)\right|=w_{l_{k}}(t) \leq 1
$$

and hence since $\varphi_{l_{k}} \in \mathfrak{N}^{+}(\mathbb{D})$, the maximum principle of V.I. Smirnov guarantees that that

$$
\left|\varphi_{l_{k}}(z)\right| \leq 1, \quad z \in \mathbb{D} .
$$

Thus, combining (3.16) and (3.17) we see that $(\gamma)$ is fulfilled.

Moreover, from (3.16) and (3.14) we get that $(\alpha)$ is satisfied.

For almost all $t \in \mathbb{T}$ with respect to $m$ we have $\left|\varphi_{l_{k}}(t)\right| \leq 1$ and, consequently, in view of (3.15), the inequality

$$
\ln ^{+}\left\|\underline{F_{k}}(t)\right\| \leq \ln ^{+}\left\|\underline{E}^{-1}(t)\right\|
$$

holds for almost all $t \in \mathbb{T}$ with respect to $m$. Hence, the family $\left(\ln ^{+}\left\|\underline{F_{k}}(t)\right\|\right)_{k \in \mathbb{N}}$ has an $m$-integrable majorant. This implies that $(\beta)$ is fulfilled.

Part (i) of Theorem 3.2 is now proved.

Before proving part (ii) of Theorem 3.2 we recall the following result (see [WM1, Lemma 3.12]).

THE GENERALIZED MINKOWSKI INEQUALITY. Let $(\Omega, \mathfrak{A}, P)$ be a probability space and let $M: \Omega \rightarrow \mathfrak{M}_{n}$ be a P-integrable matrix function with nonnegative Hermitian values. Then

$$
\ln \left[\operatorname{det}\left(\int_{\Omega} M d P\right)\right] \geq \int_{\Omega} \ln [\operatorname{det} M] d P .
$$

PROOF OF PART (ii) OF THEOREM 3.2. For $k \in \mathbb{N}$ we define $v_{k}: \mathbb{T} \rightarrow[1, \infty)$ via the rule

$$
v_{k}(t):=\left\{\begin{aligned}
\left\|\underline{E}(t) \cdot \underline{F_{k}}(t)\right\| & \text { if } \quad\left\|\underline{E}(t) \cdot \underline{F_{k}}(t)\right\| \geq 1 \\
& \text { if } \quad\left\|\underline{E}(t) \cdot \underline{F_{k}}(t)\right\|<1 .
\end{aligned}\right.
$$

For $k \in \mathbb{N}$ and $t \in \mathbb{T}$ we then have

$$
\ln \left[v_{k}(t)\right] \in[0, \infty) .
$$


Combining $(\alpha)$ and (3.19) we infer that for almost all $t \in \mathbb{T}$ with respect to $m$,

$$
\lim _{k \rightarrow \infty} \ln \left[v_{k}(t)\right]=0
$$

For $k \in \mathbb{N}$ and $t \in \mathbb{T}$ we get the inequality

$$
\ln \left[v_{k}(t)\right] \leq \ln ^{+}\|\underline{E}(t)\|+\ln ^{+}\left\|\underline{F_{k}}(t)\right\|
$$

from (3.19), which together with $(\beta)$ implies that the family $\left(\ln v_{k}\right)_{k \in \mathbb{N}}$ is uniformly $m$ integrable. Combining this fact with (3.20) and (3.21), an application of Vitali's Theorem provides

$$
\lim _{k \rightarrow \infty} \int_{\mathbb{T}} \ln \left[v_{k}(t)\right] m(d t)=0 .
$$

For $k \in \mathbb{N}$ we define $\Psi_{k}: \mathbb{D} \rightarrow \mathbb{C}$ via the formula

$$
\Psi_{k}(z):=\exp \left\{-\int_{\mathbb{T}} \ln \left[v_{k}(t)\right] \frac{t+z}{t-z} m(d t)\right\} .
$$

Therefore, in view of (3.20), we obtain the inequality

$$
\begin{aligned}
\left|\Psi_{k}(z)\right| & =\exp \left\{\Re\left[-\int_{\mathbb{T}} \ln \left[v_{k}(t)\right] \frac{t+z}{t-z} m(d t)\right]\right\} \\
& =\exp \left\{-\int_{\mathbb{T}} \ln \left[v_{k}(t)\right] \frac{1-|z|^{2}}{|t-z|^{2}} m(d t)\right\} \leq \exp \{0\}=1
\end{aligned}
$$

for $z \in \mathbb{D}$. In view of (3.21), an application of Lebesgue's dominated convergence theorem yields

$$
\lim _{k \rightarrow \infty} \Psi_{k}(z)=1
$$

for all $z \in \mathbb{D}$. For almost all $t \in \mathbb{T}$ with respect to $m$ we get from (3.24)

$$
\left|\underline{\Psi_{k}}(t)\right| \leq 1
$$

and hence, upon taking into account that formula (3.23) implies that

$$
\left|\underline{\Psi_{k}}(t)\right|=\left[v_{k}(t)\right]^{-1},
$$

we see from (3.19) and $(\alpha)$ that

$$
\lim _{k \rightarrow \infty}\left|\underline{\Psi_{k}}(t)\right|=1
$$

For $k \in \mathbb{N}$,

$$
\int_{\mathbb{T}}\left|\underline{\Psi_{k}}(t)-1\right|^{2} m(d t)=\left.\int_{\mathbb{T}} \underline{\mid \Psi_{k}}(t)\right|^{2} m(d t)-2 \Re\left[\Psi_{k}(0)\right]+1 .
$$


In view of (3.26) and (3.28), Lebesgue's dominated convergence theorem yields

$$
\lim _{k \rightarrow \infty} \int_{\mathbb{T}}\left|\underline{\Psi_{k}}(t)\right|^{2} m(d t)=m(\mathbb{T})=1 .
$$

Combining (3.25), (3.29) and (3.30) we obtain

$$
\lim _{k \rightarrow \infty} \int_{\mathbb{T}}\left|\underline{\Psi_{k}}(t)-1\right|^{2} m(d t)=0 .
$$

In view of (3.31), the F. Riesz - Fischer theorem provides a subsequence $\left(\underline{\Psi_{l_{k}}}\right)_{k \in \mathbb{N}}$ of $\left(\underline{\Psi_{k}}\right)_{k \in \mathbb{N}}$ such that

$$
\lim _{k \rightarrow \infty} \frac{\Psi_{l_{k}}}{(t)=1}
$$

for almost all $t \in \mathbb{T}$ with respect to $m$. Suppose that $k \in \mathbb{N}$ and define

$$
\Phi_{k}:=E \cdot F_{k} \cdot \Psi_{k}
$$

Then, since $E \in \mathfrak{N}_{n}^{+}(\mathbb{D}), F_{k} \in H_{n}^{\infty}(\mathbb{D})$ and (3.24) holds, we get

$$
\Phi_{k} \in \mathfrak{N}_{n}^{+}(\mathbb{D}) .
$$

For almost all $t \in \mathbb{T}$ with respect to $m$ it follows from (3.33), (3.19) and (3.27) that

$$
\left\|\underline{\Phi_{k}}(t)\right\|=\left|\underline{\Psi_{k}}(t)\right| \cdot\left\|\underline{E}(t) \cdot \underline{F_{k}}(t)\right\| \leq\left|\underline{\Psi_{k}}(t)\right| \cdot v_{k}(t)=1 .
$$

Therefore the maximum principle of V.I. Smirnov implies that

$$
\left\|\underline{\Phi_{k}}(z)\right\| \leq 1
$$

for all $z \in \mathbb{D}$. In particular,

$$
\Phi_{k} \in H_{n}^{\infty}(\mathbb{D}) .
$$

From (3.34) and (3.35) it follows that

$$
\underline{\Phi}^{\star}(t) \cdot \underline{\Phi_{k}}(t) \leq I_{n}
$$

for almost all $t \in \mathbb{T}$ with respect to $m$. Combining (3.33), $(\alpha)$ and (3.28) we get

$$
\lim _{k \rightarrow \infty} \underline{\Phi_{k}}{ }^{\star}(t) \cdot \underline{\Phi_{k}}(t)=\lim _{k \rightarrow \infty}\left|\underline{\Psi_{k}}(t)\right|^{2}\left[\underline{E}(t) \underline{F_{k}}(t)\right]^{\star}\left[\underline{E}(t) \underline{F_{k}}(t)\right]=I_{n} .
$$

From $(3.32),(3.33)$ and $(\alpha)$ we now obtain

$$
\lim _{k \rightarrow \infty} \underline{\Phi_{l_{k}}}(t)=\lim _{k \rightarrow \infty} \underline{E}(t) \cdot \underline{F_{l_{k}}}(t) \cdot \underline{\Psi_{l_{k}}}(t)=I_{n} .
$$

Using (3.37), (3.38), (3.40) and Lebesgue's dominated convergence theorem we get

$$
\lim _{k \rightarrow \infty} \Phi_{l_{k}}(0)=\lim _{k \rightarrow \infty} \int_{\mathbb{T}} \underline{\Phi_{l_{k}}}(t) m(d t)=I_{n} .
$$


Suppose that $k \in \mathbb{N}$. We define $M_{k}: \mathbb{T} \rightarrow \mathfrak{M}_{n}$ via the rule

$$
M_{k}(t):=\underline{\Phi_{k}^{*}}(t) \cdot \underline{\Phi_{k}}(t) .
$$

Then (3.42) and (3.38) imply that the inequality $0 \leq M_{k}(t) \leq I_{n}$ holds true for almost all $t \in \mathbb{T}$ with respect to $m$. Hence,

$$
0 \leq \int_{\mathbb{T}} M_{k}(t) m(d t) \leq I_{n}
$$

Now we apply the Generalized Minkowski inequality to the $M_{k}$. (Note that Lebesgue measure $m$ is a probability measure.) From (3.43) we infer first that

$$
\ln \left[\operatorname{det}\left(\int_{\mathbb{T}} M_{k}(t) m(d t)\right)\right] \leq \ln \left[\operatorname{det} I_{n}\right]=0 .
$$

Hence, (3.44) and the Generalized Minkowski inequality guarantee that

$$
\int_{\mathbb{T}} \ln \left(\operatorname{det}\left[M_{k}(t)\right]\right) m(d t) \leq 0 .
$$

Using (3.42) and (3.33) it follows that

$$
\begin{aligned}
\frac{1}{2} \ln \left(\operatorname{det}\left[M_{k}(t)\right]\right) & =\ln \left|\operatorname{det}\left[\underline{\Phi_{k}}(t)\right]\right| \\
& =\ln |\operatorname{det}[\underline{E}(t)]|+\ln \left|\operatorname{det}\left\{\left[\underline{F_{k}}(t)\right] \cdot\left[\underline{\Psi_{k}}(t)\right]\right\}\right|
\end{aligned}
$$

for almost all $t \in \mathbb{T}$ with respect to $m$. Thus, from (3.45) and (3.46) we see that

$$
\int_{\mathbb{T}} \ln |\operatorname{det}[\underline{E}(t)]| m(d t) \leq-\int_{\mathbb{T}} \ln \left|\operatorname{det}\left\{\left[\underline{F_{k}}(t)\right] \cdot\left[\underline{\Psi_{k}}(t)\right]\right\}\right| m(d t) .
$$

By assumption, $F_{k} \in H_{n}^{\infty}(\mathbb{D})$. Using (3.23) and (3.24) we see that $\Psi_{k} \in H_{n}^{\infty}(\mathbb{D})$. Thus, $F_{k} \cdot \Psi_{k} \in H_{n}^{\infty}(\mathbb{D})$ and, consequently, $\operatorname{det}\left[F_{k} \cdot \Psi_{k}\right] \in H^{\infty}(\mathbb{D})$. Now Jensen's inequality gives

$$
-\int_{\mathbb{T}} \ln \left|\operatorname{det}\left\{\left[\underline{F_{k}}(t)\right] \cdot\left[\underline{\Psi_{k}}(t)\right]\right\}\right| m(d t) \leq-\ln \left|\operatorname{det}\left\{\left[\underline{F_{k}}(0)\right] \cdot\left[\underline{\Psi_{k}}(0)\right]\right\}\right| .
$$

From (3.47) and (3.48) it now follows that

$$
\int_{\mathbb{T}} \ln |\operatorname{det}[\underline{E}(t)]| m(d t) \leq-\ln \left|\operatorname{det}\left\{\left[\underline{F_{k}}(0)\right] \cdot\left[\underline{\Psi_{k}}(0)\right]\right\}\right| .
$$

From (3.33) and (3.41) we obtain

$$
\lim _{k \rightarrow \infty} \ln \left|\operatorname{det}\left\{\left[F_{l_{k}}(0)\right] \cdot\left[\Psi_{l_{k}}(0)\right]\right\}\right|=-\ln |\operatorname{det}[E(0)]| .
$$


Combining (3.49) and (3.50) we obtain

$$
\int_{\mathbb{T}} \ln |\operatorname{det}[\underline{E}(t)]| m(d t) \leq \ln |\operatorname{det}[E(0)]| .
$$

By assumption, $E \in \mathfrak{N}_{n}^{+}(\mathbb{D})$. Thus, $\operatorname{det} E \in \mathfrak{N}^{+}(\mathbb{D})$ and Jensen's inequality yields

$$
\ln |\operatorname{det}[E(0)]| \leq \int_{\mathbb{T}} \ln |\operatorname{det}[\underline{E}(t)]| m(d t) .
$$

Hence,

$$
\int_{\mathbb{T}} \ln |\operatorname{det}[\underline{E}(t)]| m(d t)=\ln |\operatorname{det}[E(0)]| .
$$

From (3.51) and Lemma 2.1 we see that $\operatorname{det} E \in \mathfrak{E}(\mathbb{D})$. Therefore, by definition 3.1, $E \in \mathfrak{E}_{n}(\mathbb{D})$. Part (ii) of Theorem 3.2 is now proved.

THEOREM 3.3. (i) Let $E \in \mathfrak{E}_{n}(\mathbb{D})$. Then there exists a sequence $\left(F_{k}\right)_{k \in \mathbb{N}}$ from $H_{n}^{\infty}(\mathbb{D})$ with the following properties:

$(\alpha)$ For almost all $t \in \mathbb{T}$ with respect to $m, \lim _{k \rightarrow \infty} \underline{F_{k}}(t) \cdot \underline{E}(t)=I_{n}$.

( $\beta$ ) The family $\left(\ln ^{+}\left\|\underline{F_{k}}\right\|\right)_{k \in \mathbb{N}}$ is uniformly integrable with respect to $\mathrm{m}$.

$(\gamma)$ There exists a Borel subset $B_{0}$ of $\mathbb{T}$ with $m\left(B_{0}\right)=0$ such that for all

$k \in \mathbb{N}$ and all $t \in \mathbb{T} \backslash B_{0}$ the inequality $\left\|\underline{F_{k}}(t) \cdot \underline{E}(t)\right\| \leq 1$ holds.

(ii) Let $E \in \mathfrak{N}_{n}^{+}(\mathbb{D})$ be such that there exists a sequence $\left(F_{k}\right)_{k \in \mathbb{N}}$ which belongs to $H_{n}^{\infty}(\mathbb{D})$ and satisfies the above conditions $(\alpha)$ and $(\beta)$. Then $E \in \mathfrak{E}_{n}(\mathbb{D})$.

PROOF. Combine Theorem 3.2 and Remark 3.2.

It should be mentioned that Ginzburg [Gi1] obtained a multiplicative integral representation for outer functions which belong to $\mathfrak{E}_{n}(\mathbb{D})$.

\section{MATRIX-VALUED INNER FUNCTIONS}

In this section, we draw our attention to a distinguished subclass of the Schur class $\mathfrak{S}_{n \times n}($ compare Definition 2.1).

DEFINITION 4.1. Let $\Theta \in \mathfrak{S}_{n \times n}(\mathbb{D})$. Then $\Theta$ is called inner if

$$
I_{n}-\underline{\Theta}^{\star}(t) \cdot \underline{\Theta}(t)=\mathbb{O}_{n \times n}
$$

for almost all $t \in \mathbb{T}$ with respect to $m$. The class of all $n \times n$ matrix-valued inner functions will be denoted by $\mathfrak{I}_{n}(\mathbb{D})$.

REMARK 4.1. Let $\Theta \in \mathfrak{I}_{n}(\mathbb{D})$. Then obviously $\operatorname{det} \Theta \not \equiv 0$. 
REMARK 4.2. Let $\Theta \in \mathfrak{I}_{n}(\mathbb{D})$. Then $\Theta^{\top} \in \mathfrak{I}_{n}(\mathbb{D})$.

The class $\mathfrak{I}_{n}(\mathbb{D})$ contains two important subclasses, namely the so - called singular inner functions and the Blaschke-Potapov products. Now we will formulate the corresponding definitions.

DEFINITION 4.2. Let $S \in \mathfrak{I}_{n}(\mathbb{D})$. Then $S$ is called singular, if $\operatorname{det}[S(z)] \neq 0$ for all $z \in \mathbb{D}$ (or in other words if $S^{-1}$ is holomorphic in $\mathbb{D}$ ). The class of all $n \times n$ matrix-valued singular inner functions will be denoted by $\mathfrak{I}_{n, s}(\mathbb{D})$.

REMARK 4.3. If $S \in \mathfrak{I}_{n, s}(\mathbb{D})$, then $S^{-1} \in \mathfrak{N}_{n}(\mathbb{D})$, be cause $S^{-1}$ admits the representation $S^{-1}=L \cdot(\operatorname{det} S)^{-1}$ with bounded holomorphic functions $L$ and $\operatorname{det} S$.

LEMMA 4.1. Let $S \in \mathfrak{I}_{n, s}(\mathbb{D})$ be such that $S^{-1} \in \mathfrak{N}_{n}^{+}(\mathbb{D})$. Then $S$ is constant.

PROOF. Since $\underline{S}(t)$ is unitary for a.e. $t \in \mathbb{T}$ it follows that

$$
\left\|\underline{S^{-1}}(t)\right\|=1 .
$$

Therefore, by the maximum principle of V.I. Smirnov, $\left\|S^{-1}(z)\right\| \leq 1$ for all $z \in \mathbb{D}$. Since $\|S(z)\| \leq 1$ then it follows that $S(z)$ is a unitary matrix for all $z \in \mathbb{D}$. However a holomorphic matrix function with unitary values is necessarily constant (see e.g. Corollary 2.3.2 in $[\mathrm{DFK}])$.

Now we are going to define Blaschke-Potapov products. For this reason, we recall first the notion of a scalar elementary Blaschke factor. Let $a \in \mathbb{D}$. Then we define $b_{a}: \mathbb{D} \rightarrow \mathbb{C}$ via the rule

$$
b_{a}(z):=\left\{\begin{array}{lll}
\frac{|a|}{a} \cdot \frac{a-z}{1-\bar{a} z} & , \text { if } & a \in \mathbb{D} \backslash\{0\} \\
z & , \text { if } & a=0
\end{array} .\right.
$$

Assume that $P \in \mathfrak{M}_{n}$ is a non-zero orthoprojection matrix, i.e., that the conditions

$$
P^{2}=P \quad P=P^{\star}
$$

are satisfied. Then the matrix-valued function $B_{a, P}: \mathbb{D} \rightarrow \mathfrak{M}_{n}$ which is defined by

$$
B_{a, P}(z):=I_{n}+\left[b_{a}(z)-1\right] \cdot P
$$

is called the Blaschke-Potapov elementary factor associated with a and $P$. From (4.3) and (4.4) it is clear that

$$
\operatorname{det}\left[B_{a, P}\right]=\left(b_{a}\right)^{\text {rank } P} .
$$

Suppose that $\left(z_{k}\right)_{k \in I}$ is a sequence from $\mathbb{D}$ and that $\left(P_{k}\right)_{k \in I}$ is a sequence of orthoprojection matrices for which the condition

$$
\sum_{k \in I}\left(1-\left|z_{k}\right|\right) \cdot \operatorname{tr} P_{k}<+\infty
$$


is fulfilled. (The index set $I$ can be finite or infinite.) Then, according to a result due to V.P. Potapov [Pot], the product

$$
\prod_{k \in I}^{\curvearrowright} B_{z_{k}, P_{k}}(z) \quad\left(\text { resp. } \prod_{k \in I}^{\curvearrowleft} B_{z_{k}, P_{k}}(z)\right)
$$

converges for all $z \in \mathbb{D}$. (V.P. Potapov has also shown that condition (4.6) is necessary for the convergence of the product in (4.7)).

DEFINITION 4.3. Let $B: \mathbb{D} \rightarrow \mathfrak{M}_{n}$. Then $B$ is called a left (resp. right) BlaschkePotapov product if $B$ is a constant function with unitary value or if there exist a unitary matrix $V$, a set of orthoprojection matrices $\left(P_{k}\right)_{k \in I}$ and sequences $\left(z_{k}\right)_{k \in I}$ which belong to $\mathbb{D}$ such that (4.6) is satisfied and moreover the representation

$$
B=\left(\prod_{k \in I}^{\curvearrowright} B_{z_{k}, P_{k}}(z)\right) \cdot V \quad\left(\operatorname{resp} . \quad B=V \cdot\left(\prod_{k \in I}^{\curvearrowleft} B_{z_{k}, P_{k}}(z)\right)\right)
$$

is valid. The set of left (resp. right) Blaschke-Potapov products will be denoted by $\mathfrak{I}_{n, B, l}(\mathbb{D})$ $\left(\right.$ resp. $\left.\mathfrak{I}_{n, B, r}(\mathbb{D})\right)$.

We will see below that each left Blaschke-Potapov product is also a right BlaschkePotapov product and vice versa. Moreover, it will turn out that $\mathfrak{I}_{n, B, l}(\mathbb{D}) \subseteq \mathfrak{I}_{n}(\mathbb{D})$.

LEMMA 4.2. Let $A, B \in \mathfrak{C}_{n}^{*}$ be such that $A \cdot B$ is unitary. Then $A$ and $B$ are unitary too.

PROOF. Since $A, B \in \mathfrak{C}_{n}$ we have $I_{n}-A A^{\star} \geq \mathbb{O}_{n \times n}$ and $I_{n}-B B^{\star} \geq \mathbb{O}_{n \times n}$. Hence, $A\left(I_{n}-B B^{\star}\right) A^{\star} \geq \mathbb{O}_{n \times n}$. Therefore, the identity

$$
\mathbb{O}_{n \times n}=I_{n}-(A B)(A B)^{\star}=\left(I_{n}-A A^{\star}\right)+A\left(I_{n}-B B^{\star}\right) A^{\star}
$$

implies that $I_{n}-A A^{\star}=\mathbb{O}_{n \times n}$ and $A\left(I_{n}-B B^{\star}\right) A^{\star}=\mathbb{O}_{n \times n}$. Thus, $A$ is unitary. In particular, we have $\operatorname{det} A \neq 0$. This implies that $I_{n}-B B^{\star}=\mathbb{O}_{n \times n}$ and hence that $B$ is unitary too.

THEOREM 4.1. Suppose that $\Theta \in \mathfrak{I}_{n}(\mathbb{D})$.

(a) There exist functions $B \in \mathfrak{I}_{n, B, l}(\mathbb{D})\left(\right.$ resp. $\left.C \in \mathfrak{I}_{n, B, r}(\mathbb{D})\right)$ and $S \in \mathfrak{I}_{n, s}(\mathbb{D})$ (resp. $T \in \mathfrak{I}_{n, s}(\mathbb{D})$ ) such that the multiplicative representation

$$
\Theta=B \cdot S \quad(\text { resp. } \Theta=T \cdot C)
$$

holds true.

(b) Suppose that the functions $B_{1}, B_{2} \in \mathfrak{I}_{n, B, l}(\mathbb{D})$ (resp. $C_{1}, C_{2} \in \mathfrak{I}_{n, B, r}(\mathbb{D})$ ) and $S_{1}, S_{2} \in \mathfrak{I}_{n, s}(\mathbb{D})\left(\right.$ resp. $\left.T_{1}, T_{2} \in \mathfrak{I}_{n, s}(\mathbb{D})\right)$ satisfy $B_{1} S_{1}=B_{2} S_{2}=\Theta$ (resp. $T_{1} C_{1}=$ $T_{2} C_{2}=\Theta$ ). Then there exist a unitary matrix $U \in \mathfrak{M}_{n}$ (resp. $V \in \mathfrak{M}_{n}$ ) such that $B_{2}=B_{1} U$ and $S_{2}=U^{\star} S_{1}$ (resp. $C_{2}=V C_{1}$ and $T_{2}=T_{1} V^{\star}$ ) are fulfilled. 
PROOF. Theorem 4.1 is a special case of a much more general result due to V.P. Potapov [Pot]. The Potapov theory handles the case of meromorphic matrix-valued functions in $\mathbb{D}$ which have a nonidentically vanishing determinant and which are $J$-contractive where $J$ is a signature matrix (i.e. $J=J^{\star}$ and $J^{2}=I_{n}$ ). In the special case that $J=I$, V.P. Potapov's result (see [Pot] and also a series of papers by Ginzburg [Gi1] - [Gi5], $[$ GiSh] $)$ provides the existence of functions $B \in \mathfrak{I}_{n, B, l}(\mathbb{D})$ and $S \in \mathfrak{S}_{n \times n}(\mathbb{D})$ such that

$$
\Theta=B \cdot S
$$

and for all $z \in \mathbb{D}$,

$$
\operatorname{det}[S(z)] \neq 0 .
$$

Since the boundary function $\underline{\Theta}$ has unitary values almost everywhere with respect to $m$ we infer from Lemma 4.2 that the boundary functions $\underline{B}$ and $\underline{S}$ also have unitary values almost everywhere with respect to $m$. Taking into account (4.10) we obtain $S \in \mathfrak{I}_{n, s}(\mathbb{D})$. The uniqueness part goes back to V.P. Potapov [Pot] too.

LEMMA 4.3. Let $M \in \mathfrak{C}_{n}$. Then

(a) $|\operatorname{det} M| \leq 1$

(b) $|\operatorname{det} M|=1$ if and only if $M$ is unitary.

PROOF. Let $\left(l_{k}\left(M^{\star} M\right)\right)_{k=1}^{n}$ denote the system of eigenvalues of $M^{\star} M$. Then, since $M \in \mathfrak{C}_{n}, 0 \leq l_{k}\left(M^{\star} M\right) \leq 1$ for all $k \in\{1, \ldots, n\}$. Thus, as

$$
|\operatorname{det} M|^{2}=\operatorname{det}\left(M^{\star} M\right)=\prod_{k=1}^{n} l_{k}\left(M^{\star} M\right),
$$

we see that $|\operatorname{det} M| \leq 1$ with equality if and only if $l_{k}\left(M^{\star} M\right)=1$ for all $k \in\{1, \ldots, n\}$. But $l_{k}\left(M^{\star} M\right)=1$ for all $k \in\{1, \ldots, n\}$ if and only if $M^{\star} M=I_{n}$.

Now we recall a well-known characterization of Blaschke products (see e.g., Privalov [Pri, Ch.I, Sec.7.1]).

LEMMA 4.4. Let $\Theta \in \mathfrak{S}_{1 \times 1}(\mathbb{D})$. Then $\Theta$ is a Blaschke product if and only if

$$
\lim _{r \rightarrow 1-0} \int_{\mathbb{T}} \ln |\operatorname{det}[\Theta(r t)]| m(d t)=0 .
$$

THEOREM 4.2. Let $f \in \mathfrak{S}_{n \times n}(\mathbb{D})$. Then:

(a) The function $\operatorname{det} f$ belongs to $\mathfrak{S}_{1 \times 1}(\mathbb{D})$.

(b) $f \in \mathfrak{I}_{n}(\mathbb{D})$ if and only if $\operatorname{det} f \in \mathfrak{I}_{1}(\mathbb{D})$. If $f \in \mathfrak{I}_{n}(\mathbb{D})$ then $\operatorname{det} f \not \equiv 0$.

(c) $f \in \mathfrak{I}_{n, s}(\mathbb{D})$ if and only if $\operatorname{det} f \in \mathfrak{I}_{1, s}(\mathbb{D})$.

(d) The following statements are equivalent:

(i) $f \in \mathfrak{I}_{n, B, l}(\mathbb{D})$,

(ii) $f \in \mathfrak{I}_{n, B, r}(\mathbb{D})$,

(iii) $\operatorname{det} f$ is a Blaschke product.

(iv) The limit relation $\lim _{r \rightarrow 1-0} \int_{\mathbb{T}} \ln |\operatorname{det}[f(r t)]| m(d t)=0$ holds true. 
PROOF. The assertions stated in part (a) and (b) are immediate consequences of Lemma 4.3. Part (c) follows from part (a) and the definition of a singular inner function. It remains to prove part (d). From (a) and Lemma 4.4 we can immediately conclude the equivalence of statements (iii) and (iv). In view of (4.5), it is readily checked that each of the conditions (i) and (ii) implies (iii). Now suppose that (iii) holds. By virtue of part (b) we see that $f$ is an inner function. ¿From Theorem 4.1 we infer that there exist functions $B \in \mathfrak{I}_{n, B, l}(\mathbb{D})$ and $S \in \mathfrak{I}_{n, s}(\mathbb{D})$ satisfying the multiplicative decomposition $f=B \cdot S$. Hence, $\operatorname{det} f=\operatorname{det} B \cdot \operatorname{det} S$. The implication "(i) $\Rightarrow$ (iii)" which is already verified shows that $\operatorname{det} B$ is a Blaschke product. Part (c) yields that $\operatorname{det} S$ is a singular inner function. Therefore, the uniqueness part of Theorem 4.1 yields that $\operatorname{det} S$ is a constant inner function with unimodular value. Hence, we obtain from part (b) of Lemma 4.3 that the matrix $S(z)$ is unitary for each $z \in \mathbb{D}$. Since $S$ belongs to $\mathfrak{S}_{n \times n}(\mathbb{D})$, the maximum modulus principle for matrix-valued Schur functions (see e.g. [DFK,Corollary 2.3.2]) implies that $S$ is a constant function. From $f=B \cdot S$ we infer that (i) holds. The implication "(iii) $\Rightarrow$ (ii)" can be shown analogously. The theorem is proved.

For further results on matrix-valued and operator-valued inner functions we refer the reader to the monographs Helson [Hel1], Sz.-Nagy and Foias [SZNF] and Nikolskii [Nik2].

\section{INNER - OUTER FACTORIZATION}

This section is aimed at a Smirnov class generalization of the inner-outer factorization of matrix-valued functions belonging to the Hardy class $H_{n}^{2}(\mathbb{D})$.

Let us recall the following notions:

DEFINITION 5.1. The Hardy class $H_{n}^{2}(\mathbb{D})$ is the set of all matrix-valued functions $F: \mathbb{D} \rightarrow \mathfrak{M}_{n}$ which are holomorphic in $\mathbb{D}$ and satisfy

$$
\sup _{r \in[0,1)} \int_{\mathbb{T}}\|F(r t)\|^{2} m(d t)<\infty .
$$

REMARK 5.1. Obviously, $H_{n}^{\infty}(\mathbb{D}) \subseteq H_{n}^{2}(\mathbb{D}) \subseteq \mathfrak{N}_{n}^{+}(\mathbb{D})$.

REMARK 5.2. Define $\|\bullet\|_{H^{2}}: H_{n}^{2}(\mathbb{D}) \rightarrow[0, \infty)$ via

$$
F \rightarrow \sqrt{\sup _{r \in[0,1)} \int_{\mathbb{T}}\|F(r t)\|^{2} m(d t) .}
$$

Then $\left(H_{n}^{2}(\mathbb{D}),\|\bullet\|_{H^{2}}\right)$ is a complex Hilbert space.

REMARK 5.3. Let $S \in \mathfrak{S}_{n \times n}(\mathbb{D})$ be such that $\operatorname{det}\left(I_{n}+S\right)$ does not identically vanish in $\mathbb{D}$. Then $\left(I_{n}+S\right) \in \mathfrak{E}_{n}(\mathbb{D}) \cap H_{n}^{\infty}(\mathbb{D})$ (see Arov $[\operatorname{Ar1}]$, Lemma 3.1).

The definition of a matrix-valued outer function given above (see Definition 3.1) is too rough for the purposes of prediction theory of stationary sequences. For this reason, P.R. Masani [Ma1, Ma2] introduced the following notion for the space $H_{n}^{2}(\mathbb{D})$ (compare Lemma $2.2)$. 
DEFINITION 5.2. Let $E \in H_{n}^{2}(\mathbb{D})$. Then $E$ is said to be left optimal ( resp. right optimal) if $E$ has the following property: If $F \in H_{n}^{2}(\mathbb{D})$ satisfies $[\underline{F}(t)] \cdot[\underline{F}(t)]^{*}=[\underline{E}(t)] \cdot[\underline{E}(t)]^{*}$ (resp. $\left.[\underline{F}(t)]^{*} \cdot[\underline{F}(t)]=[\underline{E}(t)]^{*} \cdot[\underline{E}(t)]\right)$ then $[F(0)] \cdot[F(0)]^{*} \leq[E(0)] \cdot[E(0)]^{*}(\operatorname{resp}$. $\left.[F(0)]^{*} \cdot[F(0)] \leq[E(0)]^{*} \cdot[E(0)]\right)$.

REMARK 5.4. Let $E \in H_{n}^{2}(\mathbb{D})$. Then $E$ is left optimal if and only if $E^{\top}$ is right optimal.

This notion of optimality is closely related to the following definition which in the scalar case goes back to Beurling [Be].

DEFINITION 5.3. Let $E \in H_{n}^{2}(\mathbb{D})$. Then $E$ is called left Beurling-outer ( resp. right Beurling outer $)$ if there exists a sequence $\left(f_{k}\right)_{k \in \mathbb{N}}$ from $H_{n}^{\infty}(\mathbb{D})$ which satisfies

$$
\lim _{k \rightarrow \infty} \int_{\mathbb{T}}\left\|\underline{F_{k}}(t) \cdot \underline{E}(t)-I_{n}\right\|^{2} m(d t)=0 \quad\left(\text { resp. } \lim _{k \rightarrow \infty} \int_{\mathbb{T}}\left\|\underline{E}(t) \cdot \underline{F_{k}}(t)-I_{n}\right\|^{2} m(d t)=0\right) .
$$

The class of all $n \times n$ matrix-valued left Beurling-outer (resp. right Beurling outer) functions will be denoted by $\mathfrak{E}_{n, B, l}(\mathbb{D})$ (resp. $\mathfrak{E}_{n, B, r}(\mathbb{D})$ ).

REMARK 5.5. Let $E \in H_{n}^{2}(\mathbb{D})$. Then $E \in \mathfrak{E}_{n, B, l}(\mathbb{D})$ if and only if $E^{\top} \in \mathfrak{E}_{n, B, r}(\mathbb{D})$.

REMARK 5.6. Let $E \in H_{n}^{2}(\mathbb{D})$. Then it is readily checked that $E$ is left Beurling outer (resp. right Beurling outer) if and only if the subspace $H_{n}^{2}(\mathbb{D}) \cdot E\left(\operatorname{resp} . E \cdot H_{n}^{2}(\mathbb{D})\right)$ is dense in $\left(H_{n}^{2}(\mathbb{D}),\|\bullet\|_{H^{2}}\right)$.

REMARK 5.7. Let $E$ be a function belonging to $\mathfrak{E}_{n, B, l}(\mathbb{D})$ or $\mathfrak{E}_{n, B, r}(\mathbb{D})$. Then for all $z \in \mathbb{D}$ the relation $\operatorname{det}[E(z)] \neq 0$ holds true.

PROOF : Let us consider the case $E \in \mathfrak{E}_{n, B, r}(\mathbb{D})$. Then there exists a sequence $\left(F_{k}\right)_{k \in \mathbb{N}}$ from $H^{\infty}(\mathbb{D})$ such that

$$
\lim _{k \rightarrow \infty} \int_{\mathbb{T}}\left\|\underline{E}(t) \cdot \underline{F_{k}}(t)-I_{n}\right\|^{2} m(d t)=0
$$

From this it follows by the Poisson integral representation for $H_{n}^{2}(\mathbb{D})$ functions that

$$
\lim _{k \rightarrow \infty} E(z) \cdot F_{k}(z)=I_{n}
$$

for $z \in \mathbb{D}$ and hence that

$$
\lim _{k \rightarrow \infty} \operatorname{det}[E(z)] \cdot \operatorname{det}\left[F_{k}(z)\right]=1 .
$$

Thus, $\operatorname{det}[E(z)] \neq 0$. If $E \in \mathfrak{E}_{n, B, l}(\mathbb{D})$, then the assertion follows from Remark 5.5 and the preceding analysis.

The following result due to Masani [Ma2, Corollary 4.6] clarifies the relation between optimality and Beurling-outerness. 
THEOREM 5.1. Let $E \in H_{n}^{2}(\mathbb{D})$. Then:

(a) If $\operatorname{det} E \not \equiv 0$ and $E$ is left optimal (resp. right optimal), then $E \in \mathfrak{E}_{n, B, l}(\mathbb{D})$ (resp. $E \in \mathfrak{E}_{n, B, r}(\mathbb{D})$ ).

(b) If $E \in \mathfrak{E}_{n, B, l}(\mathbb{D})$ (resp. $E \in \mathfrak{E}_{n, B, r}(\mathbb{D})$ ), then $E$ is left optimal (resp. right optimal).

The notion of optimality is more general than the notion of Beurling - outer because it allows the functions in question to have identically vanishing determinants. In the theory of multivariate stationary stochastic processes this corresponds to the case of a singular prediction error matrix.

The following result plays a key role in the theory of holomorphic matrix-valued functions.

THEOREM 5.2. Let $F \in H_{n}^{2}(\mathbb{D})$ be such that $\operatorname{det} F \not \equiv 0$. Then:

(i) There exist functions $\Theta_{r} \in \mathfrak{I}_{n}(\mathbb{D})$ and $E_{r} \in \mathfrak{E}_{n, B, r}(\mathbb{D})$ such that the multiplicative decomposition

$$
F=\Theta_{r} \cdot E_{r}
$$

is satisfied.

(ii) Suppose that the functions $\Theta_{r 1}, \Theta_{r 2} \in \mathfrak{I}_{n}(\mathbb{D})$ and $E_{r 1}, E_{r 2} \in \mathfrak{E}_{n, B, r}(\mathbb{D})$ satisfy

$$
\Theta_{r 1} \cdot E_{r 1}=\Theta_{r 2} \cdot E_{r 2}=F \text {. }
$$

Then there exists a unitary matrix $V \in \mathfrak{M}_{n}$ such that $\Theta_{r 2}=\Theta_{r 1} \cdot V$ and $E_{r 2}=V^{\star} \cdot E_{r 1}$ are fulfilled.

(iii) There exist functions $\Theta_{l} \in \mathfrak{I}_{n}(\mathbb{D})$ and $E_{l} \in \mathfrak{E}_{n, B, l}(\mathbb{D})$ such that the multiplicative decomposition

$$
F=E_{l} \cdot \Theta_{l}
$$

is satisfied.

(iv) Suppose that the functions $\Theta_{l 1}, \Theta_{l 2} \in \mathfrak{I}_{n}(\mathbb{D})$ and $E_{l 1}, E_{l 2} \in \mathfrak{E}_{n, B, l}(\mathbb{D})$ satisfy

$$
E_{l 1} \cdot \Theta_{l 1}=E_{l 2} \cdot \Theta_{l 2}=F .
$$

Then there exists a unitary matrix $U \in \mathfrak{M}_{n}$ such that $\Theta_{l 2}=U \cdot \Theta_{l 1}$ and $E_{l 2}=E_{l 1} \cdot U^{\star}$ are fulfilled.

Theorem 5.2 was proved independently by several authors (see Masani [Ma2, 4.3, 4.4], Helson and Lowdenslager [HL2, Theorem 15], Rozanov [Roz1, Theorem 5]). The BeurlingLax-Halmos Theorem (see Beurling [Be], Lax [La], Halmos [Hal] and also Masani [Ma2, Theorem 3.8.]) which describes the structure of shift invariant left (resp. right) submodules of $H_{n}^{2}(\mathbb{D})$ lies at the heart of the proof.

THEOREM 5.3. The identities

$$
\mathfrak{E}_{n, B, l}(\mathbb{D})=\mathfrak{E}_{n, B, r}(\mathbb{D})=\mathfrak{E}_{n}(\mathbb{D}) \cap H_{n}^{2}(\mathbb{D})
$$

are valid. 
PROOF. First we show that

$$
\mathfrak{E}_{n, B, r}(\mathbb{D})=\mathfrak{E}_{n}(\mathbb{D}) \cap H_{n}^{2}(\mathbb{D}) .
$$

Our proof is based mainly on Theorem 3.2.

First assume that $E \in \mathfrak{E}_{n}(\mathbb{D}) \cap H_{n}^{2}(\mathbb{D})$. Then part (i) of Theorem 3.2 guarantees the existence of a sequence $\left(F_{k}\right)_{k \in \mathbb{N}}$ from $H_{n}^{\infty}(\mathbb{D})$ with the properties $(\alpha),(\beta)$ and $(\gamma)$ formulated there. In view of property $(\gamma)$, there exists a Borel subset $B_{0}$ of $\mathbb{T}$ with $m\left(B_{0}\right)=0$ such that for all $k \in \mathbb{N}$ and all $t \in \mathbb{T} \backslash B_{0}$ the inequality

$$
\left\|\underline{E}(t) \cdot \underline{F_{k}}(t)-I_{n}\right\| \leq\left\|\underline{E}(t) \cdot \underline{F_{k}}(t)\right\|+\left\|I_{n}\right\| \leq 2
$$

holds . In view of $(\alpha)$ and (5.1), an application of Lebesgue's dominated convergence theorem yields

$$
\lim _{k \rightarrow \infty} \int_{\mathbb{T}}\left\|\underline{E}(t) \cdot \underline{F_{k}}(t)-I_{n}\right\|^{2} m(d t)=0 .
$$

Thus, $E \in \mathfrak{E}_{n, B}(\mathbb{D})$. Hence, the inclusion

$$
\mathfrak{E}_{n}(\mathbb{D}) \cap H_{n}^{2}(\mathbb{D}) \subseteq \mathfrak{E}_{n, B}(\mathbb{D})
$$

holds true.

Now assume that $E \in \mathfrak{E}_{n, B}(\mathbb{D})$. Then Definition 5.3 implies that

$$
E \in H_{n}^{2}(\mathbb{D})
$$

We will show that $E$ satisfies the conditions $(\alpha)$ and $(\beta)$ in Theorem 3.2. In view of Definition 5.2 there exists a sequence $\left(F_{k}\right)_{k \in \mathbb{N}}$ from $H_{n}^{\infty}(\mathbb{D})$ for which

$$
\lim _{k \rightarrow \infty} \int_{\mathbb{T}}\left\|\underline{E}(t) \cdot \underline{F_{k}}(t)-I_{n}\right\|^{2} m(d t)=0 .
$$

Obviously, for $k \in \mathbb{N}$ and $t \in \mathbb{T}$ the inequality

$$
0 \leq \ln ^{+}\left\|\underline{E}(t) \cdot \underline{F_{k}}(t)\right\| \leq\left\|\underline{E}(t) \cdot \underline{F_{k}}(t)-I_{n}\right\|
$$

holds true. From (5.4) and (5.5) it then follows that

$$
\lim _{k \rightarrow \infty} \int_{\mathbb{T}} \ln ^{+}\left\|\underline{E}(t) \cdot \underline{F_{k}}(t)\right\| m(d t)=0 .
$$

Hence, the family $\left(\ln ^{+}\left\|\underline{E} \cdot \underline{F_{k}}\right\|\right)_{k \in \mathbb{N}}$ is uniformly $m$ - integrable. In view of Remark 5.7 we see that $\operatorname{det}[E(z)] \neq 0$ for all $z \in \mathbb{D}$. Since $E \in H_{n}^{2}(\mathbb{D}) \subseteq \mathfrak{N}_{n}(\mathbb{D})$ we now obtain $E^{-1} \in \mathfrak{N}_{n}(\mathbb{D})$. Hence, $\ln \left\|\underline{E}^{-1}\right\|=\ln \left\|\underline{E^{-1}}\right\|$ is $m$-integrable. Clearly, for $k \in \mathbb{N}$ and $t \in \mathbb{T}$ the inequality

$$
\ln ^{+}\left\|\underline{F_{k}}(t)\right\| \leq \ln ^{+}\left\|\underline{E}(t) \cdot \underline{F_{k}}(t)\right\|+\ln ^{+}\left\|[\underline{E}(t)]^{-1}\right\|
$$


holds true. Since the family $\left(\ln ^{+}\left\|\underline{E} \cdot \underline{F_{k}}\right\|\right)_{k \in \mathbb{N}}$ is uniformly $m$-integrable and since $\ln \left\|\underline{E}^{-1}\right\|$ is $m$-integrable it follows from (5.6) that the family $\left(\ln ^{+}\left\|\underline{F_{k}}\right\|\right)_{k \in \mathbb{N}}$ is uniformly $m$ integrable. Taking into account (5.4), the Theorem of F. Riesz - Fischer provides the existence of a subsequence $\left(F_{l_{k}}\right)_{k \in \mathbb{N}}$ of $\left(F_{k}\right)_{k \in \mathbb{N}}$ such that

$$
\lim _{k \rightarrow \infty} \underline{E}(t) \cdot \underline{F_{l_{k}}}(t)=I_{n}
$$

for $m$-almost all $t \in \mathbb{T}$. Since the family $\left(\ln ^{+}\left\|F_{l_{k}}\right\|\right)_{k \in \mathbb{N}}$ is also uniformly $m$-integrable the conditions $(\alpha)$ and $(\beta)$ in Theorem 3.2 are satisfied for the sequence $\left(F_{l_{k}}\right)_{k \in \mathbb{N}}$. Thus, part (ii) of Theorem 3.2 implies that

$$
E \in \mathfrak{E}_{n}(\mathbb{D}) .
$$

From $(5.3)$ and $(5.7)$ we obtain $\mathfrak{E}_{n, B}(\mathbb{D}) \subseteq \mathfrak{E}_{n}(\mathbb{D}) \cap H_{n}^{2}(\mathbb{D})$.

An application of (5.2) shows that

$$
\mathfrak{E}_{n, B, r}(\mathbb{D})=\mathfrak{E}_{n}(\mathbb{D}) \cap H_{n}^{2}(\mathbb{D}) .
$$

From (5.8) and Remarks 3.2 and 5.5 we then get

$$
\mathfrak{E}_{n, B, l}(\mathbb{D})=\mathfrak{E}_{n}(\mathbb{D}) \cap H_{n}^{2}(\mathbb{D}) .
$$

Thus, the theorem is proved.

THEOREM 5.4. (Inner - outer factorization in the Smirnov class $\mathfrak{N}_{n}^{+}(\mathbb{D})$ ). Let $F \in \mathfrak{N}_{n}^{+}(\mathbb{D})$ be such that $\operatorname{det} F \not \equiv 0$. Then:

(i) There exist functions $\Theta_{r} \in \mathfrak{I}_{n}(\mathbb{D})$ and $E_{r} \in \mathfrak{E}_{n}(\mathbb{D})$ such that

$$
F=\Theta_{r} \cdot E_{r} .
$$

(ii) Suppose that the functions $\Theta_{r 1}, \Theta_{r 2} \in \mathfrak{I}_{n}(\mathbb{D})$ and $E_{r 1}, E_{r 2} \in \mathfrak{E}_{n}(\mathbb{D})$ satisfy

$$
\Theta_{r 1} \cdot E_{r 1}=\Theta_{r 2} \cdot E_{r 2}=F
$$

Then there exists a unitary matrix $V \in \mathfrak{M}_{n}$ such that $\Theta_{r 2}=\Theta_{r 1} \cdot V$ and $E_{r 2}=V^{\star} \cdot E_{r 1}$.

(iii) There exist functions $\Theta_{l} \in \mathfrak{I}_{n}(\mathbb{D})$ and $E_{l} \in \mathfrak{E}_{n}(\mathbb{D})$ such that

$$
F=E_{l} \cdot \Theta_{l} .
$$

(iv) Suppose that the functions $\Theta_{l 1}, \Theta_{l 2} \in \mathfrak{I}_{n}(\mathbb{D})$ and $E_{l 1}, E_{l 2} \in \mathfrak{E}_{n}(\mathbb{D})$ satisfy

$$
E_{l 1} \cdot \Theta_{r 1}=E_{l 2} \cdot \Theta_{r 2}=F \text {. }
$$

Then there exists a unitary matrix $U \in \mathfrak{M}_{n}$ such that $\Theta_{l 2}=U \cdot \Theta_{l 1}$ and $E_{l 2}=E_{l 1} \cdot U^{\star}$.

PROOF. We derive these results from Theorem 5.2.

(i) In view of Lemma 2.4 there exist functions $d \in \mathfrak{E}(\mathbb{D})$ and $\Phi \in \mathfrak{S}_{n \times n}(\mathbb{D})$ such that

$$
F=\frac{1}{d} \cdot \Phi
$$


Since $\operatorname{det} F \not \equiv 0$, it follows from $(5.9)$ that $\operatorname{det} \Phi \not \equiv 0$. Thus as $\mathfrak{S}_{n \times n}(\mathbb{D}) \subseteq H_{n}^{2}(\mathbb{D})$, Theorem 5.2 ensures the existence of functions $\Theta_{r} \in \mathfrak{I}_{n}(\mathbb{D})$ and $E_{r, B} \in \mathfrak{E}_{n, B}(\mathbb{D})$ such that

$$
\Phi=\Theta_{r} \cdot E_{r, B}
$$

We set

$$
E:=d \cdot E_{r, B} .
$$

According to Theorem 5.3 it follows that $E_{r, B} \in \mathfrak{E}_{n}(\mathbb{D})$. Since $d \in \mathfrak{E}(\mathbb{D})$ we get $E \in \mathfrak{E}_{n}(\mathbb{D})$ from (5.11). Thus (i) is proved.

(ii) The factorizations $F=\Theta_{r 1} \cdot E_{r 1}=\Theta_{r 2} \cdot E_{r 2}$ yield the factorizations

$$
\Theta_{r 1} \cdot E_{r 1, B}=\Theta_{r 2} \cdot E_{r 2, B}=\Phi
$$

upon setting $E_{r 1, B}:=d \cdot E_{r 1}, E_{r 2, B}:=d \cdot E_{r 2}$ and invoking (5.9).

From $\Phi \in \mathfrak{S}_{n \times n}(\mathbb{D}),(5.12)$ and its definition it is clear that

$$
E_{r 1, B}, E_{r 2, B} \in \mathfrak{E}_{n}(\mathbb{D}) \cap \mathfrak{S}_{n \times n}(\mathbb{D}) .
$$

Thus, from Theorem 3.2 we get $E_{r 1, B}, E_{r 2, B} \in \mathfrak{E}_{n, B}(\mathbb{D})$. Now part (ii) of Theorem 5.2 provides the existence of a unitary matrix satisfying $\Theta_{r 2}=\Theta_{r 1} \cdot V$ and $E_{r 2, B}=V^{\star} \cdot E_{r 1, B}$. Hence,

$$
E_{r 2}=\frac{1}{d} \cdot E_{r 2, B}=\frac{1}{d} \cdot V^{\star} \cdot E_{r 1, B}=V^{\star} \cdot E_{r 1} .
$$

Thus, (ii) is proved.

Assertions (iii) and (iv) can be established analogously.

COROLLARY 5.1. Let $F \in \mathfrak{N}_{n}^{+}(\mathbb{D})$ be such that $\operatorname{det} F \not \equiv 0$. Then there exist functions $B_{1} \in \mathfrak{I}_{n, B, l}(\mathbb{D}), S_{1} \in \mathfrak{I}_{n, s}(\mathbb{D})$ and $E_{1} \in \mathfrak{E}_{n}(\mathbb{D})$ (resp. $B_{2} \in \mathfrak{I}_{n, B, r}(\mathbb{D}), S_{2} \in \mathfrak{I}_{n, s}(\mathbb{D})$ and $\left.E_{2} \in \mathfrak{E}_{n}(\mathbb{D})\right)$ such that

$$
F=B_{1} \cdot S_{1} \cdot E_{1} \quad\left(\operatorname{resp} . F=E_{2} \cdot S_{2} \cdot B_{2}\right)
$$

PROOF. The assertion follows immediately by combining Theorem 4.1 and Theorem 5.4.

It should be mentioned that using deep results and methods of V. Potapov [Pot] an alternate approach to Theorem 5.4 and Corollary 5.1 was presented by J.P. Ginzburg [Gi1]. His result contains also a multiplicative integral representation for the outer factor and the singular inner component.

The following theorem provides a useful characterization of the case that the inner component in the inner - outer factorization of a given function from $\mathfrak{N}_{n}^{+}(\mathbb{D})$ is a BlaschkePotapov product.

THEOREM 5.5. Let $F \in \mathfrak{N}_{n}^{+}(\mathbb{D})$ be such that $\operatorname{det} F \not \equiv 0$. Suppose that the functions $\Theta_{r}, \Theta_{l} \in \mathfrak{I}_{n}(\mathbb{D})$ and $E_{r}, E_{l} \in \mathfrak{E}_{n}(\mathbb{D})$ satisfy $\Theta_{r} \cdot E_{r}=E_{l} \cdot \Theta_{l}=F$.

Then the following statements are equivalent:

(i) $\quad \Theta_{r} \in \mathfrak{I}_{n, B, r}(\mathbb{D})$ 


$$
\begin{array}{ll}
\text { (ii) } & \Theta_{l} \in \mathfrak{I}_{n, B, l}(\mathbb{D}) \\
\text { (iii) } & \lim _{s \rightarrow 1-0} \int_{\mathbb{T}} \ln |\operatorname{det}[F(s t)]| m(d t)=\int_{\mathbb{T}} \ln |\underline{F}(t)| m(d t) .
\end{array}
$$

PROOF. In view of the fact that $E_{r}, E_{l} \in \mathfrak{E}_{n}(\mathbb{D})$, the functions $\operatorname{det} E_{r}$ and $\operatorname{det} E_{l}$ are outer. Moreover, since $\Theta_{r}, \Theta_{l} \in \mathfrak{I}_{n}(\mathbb{D})$, part (b) of Theorem 4.2 implies that the functions $\operatorname{det} \Theta_{r}$, det $\Theta_{l}$ are inner. From part (d) of Theorem 4.2 it follows that (i) (resp. (ii)) holds if and only if $\operatorname{det} \Theta_{r}$ (resp. $\operatorname{det} \Theta_{l}$ ) is a Blaschke product. According to Lemma 4.4 this is equivalent to

$$
\lim _{s \rightarrow 1-0} \int_{\mathbb{T}} \ln \left|\operatorname{det}\left[\Theta_{r}(s t)\right]\right| m(d t)=0
$$

(resp.

$$
\left.\lim _{s \rightarrow 1-0} \int_{\mathbb{T}} \ln \left|\operatorname{det}\left[\Theta_{l}(s t)\right]\right| m(d t)=0\right) .
$$

From the multiplicative decomposition $F=\Theta_{r} \cdot E_{r}$ (resp. $F=E_{l} \cdot \Theta_{l}$ ) it follows immediately that (5.13) (resp. (5.14)) is equivalent to (iii).

Thus, the statements (i) - (iii) are equivalent.

REMARK 5.8. It is instructive to compare statement (iii) in Theorem 5.5 with the inequality (1.15) which is fulfilled for an arbitrary function $F$ from $\mathfrak{N}_{n}^{+}(\mathbb{D})$.

\section{AN ANALOGUE OF FROSTMAN'S THEOREM FOR MATRIX FUNCTIONS OF THE SMIRNOV CLASS}

Let $f$ be a nonconstant function from the Smirnov class $\mathfrak{N}^{+}(\mathbb{D})$. For $\lambda \in \mathbb{C}$ the function

$$
f_{\lambda}:=f-\lambda
$$

clearly belongs to $\mathfrak{N}^{+}(\mathbb{D})$ too. Thus, there exists an inner function $\theta_{\lambda}$ and an outer function $e_{\lambda}$ such that

$$
f_{\lambda}=\theta_{\lambda} \cdot e_{\lambda}
$$

It will turn out that in some sense "the typical situation" corresponds to the case that the inner function $\theta_{\lambda}$ in (6.2) is a Blaschke product. The set of all $\lambda \in \mathbb{C}$ for which $\theta_{\lambda}$ is not a Blaschke product is very thin. (A remarkable result of this type goes back to Frostman $[\mathrm{Fr}]$.$) The corresponding notion of thinness can be formulated in terms of potential theory.$ For this reason, now we recall some notions of potential theory.

Suppose that $\nu$ is a nonnegative Borel measure with compact support. For all $\xi \in \mathbb{C}$ the integral

$$
U^{(\nu)}(\xi):=\int_{\mathbb{C}} \ln |\xi-\lambda| \nu(d \lambda)
$$

is then well-defined and takes its values in $[-\infty, \infty)$. The function $U^{(\nu)}: \mathbb{C} \rightarrow[-\infty, \infty)$ is called the logarithmic potential of $\nu$. A Borel measure $\nu$ on $\mathbb{C}$ is said to be nontrivial if it is not the zero measure. If $K$ is a Borel subset of $\mathbb{C}$, the Borel measure $\nu$ is said to be 
concentrated on $K$ if $\nu(\mathbb{C} \backslash K)=0$. By definition, a Borel subset $K$ on $\mathbb{C}$ is called thin if for each nontrivial Borel measure $\nu$ which is concentrated on $K$ the associated logarithmic potential $U^{(\nu)}$ is not bounded from below, or in other words if

$$
\inf _{\xi \in \mathbb{C}} U^{(\nu)}(\xi)=-\infty .
$$

If $K$ is not thin, then there exists a nontrivial Borel measure $\nu$ which is concentrated on $K$ and satisfies

$$
\inf _{\xi \in \mathbb{C}} U^{(\nu)}(\xi)>-\infty .
$$

The notion of logarithmic capacity is introduced in potential theory. More precisely, this means that with each Borel subset $K$ of $\mathbb{C}$ there is associated a nonnegative number cap $K$ which is called the logarithmic capacity of $K$. It turns out that a Borel subset $K$ of $\mathbb{C}$ is thin if and only if $\operatorname{cap} K=0$. In other words, if $\operatorname{cap} K>0$, then there exists a nontrivial Borel measure $\nu$ which is concentrated on $K$ and satisfies condition (6.4). If cap $K>0$, then amongst all the nontrivial Borel measures $\nu$ which are concentrated on $K$ and satisfy (6.4) there is a distinguished probability measure $\nu_{K}$, the so-called equilibrium measure of $K$. This measure $\nu_{K}$ is a solution of several natural extremal problems. (If cap $K=0$ the equilibrium measure is not defined.)

The logarithmic potential is not always continuous on $\mathbb{C}$ but only upper semicontinuous on $\mathbb{C}$. More precisely, for all $\xi \in \mathbb{C}$,

$$
\varlimsup_{\xi^{\prime} \rightarrow \xi} U^{(\nu)}\left(\xi^{\prime}\right) \leq U^{(\nu)}(\xi)
$$

Although it is bounded below, the logarithmic potential of the equilibrium measure need not be continuous on $\mathbb{C}$. If the set $K$ is "bad" there are so-called irregular points. Nevertheless it can be proved (see de la Vallée Poussin [LVP2], [LVP3]) that if cap $K>0$, then there exists a nontrivial nonnegative measure which is concentrated on $K$ and for which the associated logarithmic potential is continuous on $\mathbb{C}$ (as already mentioned, the equilibrium measure $\nu_{K}$ can not generally be used for this purpose). We will not enter into such detailed and rather delicate potential - theoretical considerations. To avoid them we give the following definition.

DEFINITION 6.1. A bounded Borel subset $K$ of $\mathbb{C}$ is said to have positive logarithmic capacity if there exists a nontrivial Borel measure $\nu$ which is concentrated on $K$ and for which the associated logarithmic potential is continuous on $\mathbb{C}$.

Clearly, if $K_{1} \subseteq K_{2}$ and $K_{1}$ is a set of positive logarithmic capacity, then $K_{2}$ is also a set of positive logarithmic capacity.

LEMMA ON THE CAPACITY OF AN INTERVAL. Every interval of the complex plane is a set of positive logarithmic capacity.

PROOF. Without loss of generality we can assume that the considered interval is a subinterval $(\alpha, \beta)$ of the real axis where $-\infty<\alpha<\beta<\infty$. Now we take for $\nu$ the restriction of one dimensional Lebesgue measure to this interval $(\alpha, \beta)$. The function $U^{(\nu)}: \mathbb{C} \rightarrow[-\infty, \infty)$ which is defined by the rule

$$
\xi \rightarrow \int_{(\alpha, \beta)} \ln |\xi-\lambda| \nu(d \lambda)
$$


is continuous in $\mathbb{C}$. This can be checked in several ways, e.g. one can compute explicitly and then obtain the continuity of $U^{(\nu)}$ by direct estimates.

W. Rudin [Ru1] (see also section 3.6 of the monograph [Ru2]) proved the following fact which generalizes Frostman's original result:

Let $f \in \mathfrak{N}^{+}(\mathbb{D})$ with $f \not \equiv 0$ and let $K$ be some bounded Borel subset of $\mathbb{C}$ with positive logarithmic capacity. Then there exist a $\lambda \in K$ such that the inner factor in the multiplicative decomposition (6.2) is a Blaschke product. (Indeed, W. Rudin obtained a more general result which is formulated for the Smirnov class $\mathfrak{N}^{+}\left(\mathbb{D}^{p}\right)$ in the polydisc $\mathbb{D}^{p}$. This class is a natural analogue of $\mathfrak{N}^{+}(\mathbb{D})$ and coincides with it in the case $p=1$.) It should be mentioned that S.A. Vinogradov [Vin] independently obtained such a generalization of Frostman's theorem too.

REMARK 6.1. Let $F \in \mathfrak{N}_{n}^{+}(\mathbb{D})$ and define $F_{\lambda}:=F-\lambda \cdot I_{n}$ for $\lambda \in \mathbb{C}$. Then the set $M_{F}:=\left\{\lambda \in \mathbb{C}: \operatorname{det}\left(F_{\lambda}\right) \equiv 0\right\}$ is finite.

Now we formulate our main result.

THEOREM 6.1. Let $F \in \mathfrak{N}_{n}^{+}(\mathbb{D})$. Assume that for $\lambda \in \mathbb{C} \backslash M_{F}$ the functions $\Theta_{\lambda, r} \in$ $\mathfrak{I}_{n}(\mathbb{D})$ and $E_{\lambda, r} \in \mathfrak{E}_{n}(\mathbb{D})$ are factors in the multiplicative decomposition

$$
F_{\lambda}=\Theta_{\lambda, r} \cdot E_{\lambda, r} .
$$

Suppose that $K$ is a bounded Borel subset of $\mathbb{C}$ with positive logarithmic capacity. Then there exists a point $\lambda \in K \cap\left(\mathbb{C} \backslash M_{F}\right)$ for which $\Theta_{\lambda, r}$ is a Blaschke-Potapov product.

COROLLARY 6.1. The set of all $\lambda \in \mathbb{C} \backslash M_{F}$ for which $\Theta_{\lambda, r}$ is a Blaschke-Potapov product is dense in $\mathbb{C}$.

PROOF : Combine Theorem 6.1 and the Lemma on the capacity of an interval.

In order to to follow the strategy of W. Rudin's proof we shell need to introduce a number of classes of scalar functions of several variables.

DEFINITION 6.2. A function $\sigma: \mathbb{C}^{n} \rightarrow \mathbb{R}$ is called symmetric if for all permutations

$$
\begin{array}{r}
\left(\begin{array}{ccc}
1 & \ldots & n \\
i_{1} & \ldots & i_{n}
\end{array}\right) \text { and all } x=\left(x_{1}, \ldots, x_{n}\right)^{\top} \in \mathbb{C}^{n} \text { the relation } \\
\sigma\left(\left(x_{i_{1}}, \ldots, x_{i_{n}}\right)^{\top}\right)=\sigma\left(\left(x_{1}, \ldots, x_{n}\right)^{\top}\right)
\end{array}
$$

is valid.

In view of Definition 6.2 the following object is well-defined.

DEFINITION 6.3. Let $\sigma: \mathbb{C}^{n} \rightarrow \mathbb{R}$ be a symmetric function. Then the function $\varphi_{\sigma}: \mathfrak{M}_{n} \rightarrow \mathbb{R}$ which is defined by the rule

$$
A \rightarrow \sigma\left(\left(l_{1}(A), \ldots, l_{n}(A)\right)^{\top}\right),
$$

where $\left(l_{j}(A)\right)_{j=1}^{n}$ are the roots of the characteristic polynomial of $A$ (taking into account their algebraic multiplicities), is called the function of matrix argument which is generated 
by the symmetric function $\sigma$.

LEMMA 6.1. Suppose that $\sigma: \mathbb{C}^{n} \rightarrow \mathbb{R}$ is a continuous symmetric function. Then $\varphi_{\sigma}$ is a continuous function.

PROOF. The lemma is an immediate consequence of Theorem 5.1 from Chapter II in Kato's monograph [Ka]. (See there especially formula (5.3) and the text following it.)

If the symmetric function $\sigma: \mathbb{C}^{n} \rightarrow \mathbb{R}$ is a polynomial or a rational function in $n$ variables $x_{1}, \ldots, x_{n}$, then it can be expressed as a polynomial or a rational function of the elementary symmetric functions. In this case the function $\varphi_{\sigma}$ is a polynomial or a rational function of the elements of the matrix variable.

We introduce now a potential of the matrix argument. Roughly speaking, we insert a matrix argument in formula (6.3) instead of the complex variable.

REMARK 6.2. Suppose that $A \in \mathfrak{M}_{n}$. Then the function $h_{A}: \mathbb{C} \rightarrow \mathbb{R}$ which is defined by $\lambda \rightarrow\left|\operatorname{det}\left[A-\lambda I_{n}\right]\right|$ is continuous. Hence, the function $\ln h_{A}$ is continuous and locally bounded above. If $\nu$ is a finite Borel measure on $\mathbb{C}$ with compact support, then the function $\Phi^{(\nu)}: \mathfrak{M}_{n} \rightarrow[-\infty, \infty)$ with

$$
\Phi^{(\nu)}(A):=\int_{\mathbb{C}} \ln \left|\operatorname{det}\left(A-\lambda I_{n}\right)\right| \nu(d \lambda)
$$

is well-defined.

DEFINITION 6.4. Suppose that $\nu$ is a finite Borel measure on $\mathbb{C}$ with compact support. Then the function $\Phi^{(\nu)}: \mathfrak{M}_{n} \rightarrow[-\infty, \infty)$ which is defined by (6.5) is called the potential of the matrix argument associated with $\nu$.

Assume that $\nu$ is a finite Borel measure on $\mathbb{C}$ with compact support. Let $U^{(\nu)}$ denote the logarithmic potential of $\nu$. Let $A \in \mathfrak{M}_{n}$ and let $\left(l_{k}(A)\right)_{k=1}^{n}$ be roots of the characteristic polynomial of $A$. For $\lambda \in \mathbb{C}$ we then have

$$
\ln \left|\operatorname{det}\left(A-\lambda I_{n}\right)\right|=\sum_{k=1}^{n} \ln \left|l_{k}(A)-\lambda\right| .
$$

Hence, upon taking (6.3) into account we get

$$
\Phi^{(\nu)}(A)=\sum_{k=1}^{n} U^{(\nu)}\left(l_{k}(A)\right) .
$$

We define $\sigma^{(\nu)}: \mathbb{C}_{n} \rightarrow[-\infty, \infty)$ via

$$
\left(\begin{array}{c}
x_{1} \\
\vdots \\
x_{n}
\end{array}\right) \rightarrow \sum_{k=1}^{n} U^{(\nu)}\left(x_{k}\right) .
$$

Obviously, the function $\sigma^{(\nu)}$ is symmetric. From Definition 6.3, (6.7) and (6.8) we infer that

$$
\Phi^{(\nu)}(A)=\varphi_{\sigma^{(\nu)}}(A) .
$$


LEMMA 6.2. Suppose that $\nu$ is a finite Borel measure on $\mathbb{C}$ with compact support such that the associated logarithmic potential $U^{(\nu)}$ is continuous on $\mathbb{C}$. Then the function $\Phi^{(\nu)}: \mathfrak{M}_{n} \rightarrow[-\infty, \infty)$ which is defined by (6.5) is continuous on $\mathfrak{M}_{n}$.

PROOF. Indeed, from (6.8) it follows that $\sigma^{(\nu)}$ is a continuous function on $\mathbb{C}^{n}$. Then in view of (6.9) and Lemma 6.1 the assertion follows.

DEFINITION 6.5. Let $\nu$ be a finite Borel measure on $\mathbb{C}$ with compact support. Then the functions $\Phi_{+}^{(\nu)}: \mathfrak{M}_{n} \rightarrow[0, \infty)$ and $\Phi_{-}^{(\nu)}: \mathfrak{M}_{n} \rightarrow[0, \infty)$ are defined via the formulas

$$
\Phi_{+}^{(\nu)}(A):=\int_{\mathbb{C}} \ln ^{+}\left|\operatorname{det}\left(A-\lambda I_{n}\right)\right| \nu(d \lambda)
$$

and

$$
\Phi_{-}^{(\nu)}(A):=\int_{\mathbb{C}} \ln ^{-}\left|\operatorname{det}\left(A-\lambda I_{n}\right)\right| \nu(d \lambda),
$$

respectively.

LEMMA 6.3. Suppose that $\nu$ is a finite Borel measure on $\mathbb{C}$ with compact support. Then the function $\Phi_{+}^{(\nu)}$ defined by (6.10) is continuous on $\mathfrak{M}_{n}$.

PROOF. The function $f: \mathfrak{M}_{n} \times \mathbb{C} \rightarrow[0, \infty)$ which is defined by

$$
f(A, \lambda):=\left|\operatorname{det}\left(A-\lambda I_{n}\right)\right|
$$

is continuous on $\mathfrak{M}_{n} \times \mathbb{C}$. Since the function $\ln ^{+}:=\max \{\ln , 0\}$ is continuous on $[0, \infty)$ the composition mapping $\ln ^{+} f$ is continuous on $\mathfrak{M}_{n} \times \mathbb{C}$. From this we infer that the function $\Phi_{+}^{(\nu)}$ is continuous on $\mathfrak{M}_{n}$.

LEMMA 6.4. Suppose that $\nu$ is a finite Borel measure on $\mathbb{C}$ with compact support such that the associated logarithmic potential $U^{(\nu)}$ is continuous on $\mathbb{C}$. Then the function $\Phi_{-}^{(\nu)}$ which is defined by (6.11) is continuous on $\mathfrak{M}_{n}$; it is also bounded:

$$
\sup _{A \in \mathfrak{M}_{n}} \Phi_{-}^{(\nu)}(A)<+\infty .
$$

PROOF. From Definitions 6.4 and 6.5 we get the identity

$$
\Phi^{(\nu)}=\Phi_{+}^{(\nu)}-\Phi_{-}^{(\nu)} .
$$

In view of Lemma 6.2 the function $\Phi^{(\nu)}$ is continuous whereas Lemma 6.3 provides the continuity of $\Phi_{+}^{(\nu)}$. Thus, (6.13) shows the continuity of $\Phi_{-}^{(\nu)}$. We define the functions $U_{+}^{(\nu)}: \mathbb{C} \rightarrow[0, \infty)$ and $U_{-}^{(\nu)}: \mathbb{C} \rightarrow[0, \infty)$ by

$$
U_{+}^{(\nu)}(\xi):=\int_{\mathbb{C}} \ln ^{+}|\xi-\lambda| \nu(d \lambda)
$$

and

$$
U_{-}^{(\nu)}(\xi):=\int_{\mathbb{C}} \ln ^{-}|\xi-\lambda| \nu(d \lambda) .
$$


Combining (6.3), (6.14) and (6.15) we see that

$$
U^{(\nu)}=U_{+}^{(\nu)}-U_{-}^{(\nu)}
$$

Since the function $U^{(\nu)}$ is continuous by assumption and since the function $U_{+}^{(\nu)}$ is always continuous (by Lemma 6.3 with $n=1$ ) the continuity of $U_{-}^{(\nu)}$ follows from (6.16). If $\left(r_{k}\right)_{k=1}^{n}$ is a sequence from $[0, \infty)$, then clearly

$$
\ln ^{-}\left(\prod_{k=1}^{n} r_{k}\right) \leq \sum_{k=1}^{n} \ln ^{-} r_{k} .
$$

Let $A \in \mathfrak{M}_{n}$ and let $\left(l_{k}(A)\right)_{k=1}^{n}$ be the roots of the characteristic polynomial of $A$. In view of (6.6) we get

$$
\ln ^{-}\left|\operatorname{det}\left(A-\lambda I_{n}\right)\right|=\ln ^{-}\left(\prod_{k=1}^{n}\left|l_{k}(A)-\lambda\right|\right) .
$$

From (6.17), (6.18) and (6.15) we infer that

$$
\Phi_{-}^{(\nu)}(A) \leq \sum_{k=1}^{n} U_{-}^{(\nu)}\left(l_{k}(A)\right) .
$$

Hence,

$$
\sup _{A \in \mathfrak{M}_{n}} \Phi_{-}^{(\nu)}(A) \leq n \cdot \sup _{\xi \in \mathbb{C}} U_{-}^{(\nu)}(\xi) .
$$

Now it remains to prove that our assumptions ensure that

$$
\sup _{\xi \in \mathbb{C}} U_{-}^{(\nu)}(\xi)<\infty
$$

is fulfilled. If $\xi \in \mathbb{C}$ satisfies

$$
|\xi| \geq 1+\sup _{\lambda \in \operatorname{supp} \nu}|\lambda|
$$

then using (6.15) we see that

$$
U_{-}^{(\nu)}(\xi)=0 .
$$

Now the continuity of $U_{-}^{(\nu)},(6.21),(6.22)$ and a classical theorem due to Weierstrass yield (6.20). The lemma is proved.

REMARK 6.3. If $a, b \in[0, \infty)$, then

$$
\ln ^{+}(a+b) \leq \ln ^{+} a+\ln ^{+} b+\ln 2 .
$$

REMARK 6.4. Let $A \in \mathfrak{M}_{n}$. Then $|\operatorname{det} A| \leq\|A\|^{n}$.

REMARK 6.5. Let $A \in \mathfrak{M}_{n}$ and $\lambda \in \mathbb{C}$. Then

$$
\ln ^{+}\left|\operatorname{det}\left[A-\lambda I_{n}\right]\right| \leq n \cdot\left[\ln ^{+}\|A\|+\ln ^{+}|\lambda|+\ln 2\right] .
$$


Indeed, using remarks 6.4 and 6.3 we obtain

$$
\begin{aligned}
\ln ^{+}\left|\operatorname{det}\left[A-\lambda I_{n}\right]\right| & \leq \ln ^{+}\left[\left\|A-\lambda I_{n}\right\|^{n}\right]=n \cdot \ln ^{+}\left[\left\|A-\lambda I_{n}\right\|\right] \\
& \leq n \cdot \ln ^{+}\left[\|A\|+\left\|\lambda I_{n}\right\|\right]=n \cdot \ln ^{+}[\|A\|+|\lambda|] \\
& \leq n \cdot\left[\ln ^{+}\|A\|+\ln ^{+}|\lambda|+\ln 2\right] .
\end{aligned}
$$

PROOF OF THEOREM 6.1. Let $\lambda \in \mathbb{C}$. For $r \in[0,1)$ we define

$$
v_{r}(\lambda):=\int_{\mathbb{T}} \ln \left|\operatorname{det}\left[F(r t)-\lambda I_{n}\right]\right| m(d t) .
$$

Assume that $r_{1}, r_{2} \in[0,1)$ satisfy $r_{1} \leq r_{2}$. Since the function $\operatorname{det}\left[F-\lambda I_{n}\right]$ is holomorphic we get $v_{r_{1}}(\lambda) \leq v_{r_{2}}(\lambda)$. Thus, the limit

$$
v_{1-0}(\lambda):=\lim _{r \rightarrow 1-0} v_{r}(\lambda)
$$

exists. Define

$$
v(\lambda):=\int_{\mathbb{T}} \ln \left|\operatorname{det}\left[\underline{F}(t)-\lambda I_{n}\right]\right| m(d t) .
$$

If we apply inequality (1.15) to the function $F-\lambda I_{n}$, then using (6.23) - (6.25) we obtain

$$
v_{1-0}(\lambda) \leq v(\lambda) .
$$

According to Theorem 5.5, equality holds in (6.26) for those and only those $\lambda \in \mathbb{C} \backslash M_{F}$ for which the inner factor $\Theta_{\lambda, r}$ is a Blaschke-Potapov product. Consequently, Theorem 5.5 reduces the question which is discussed in Theorem 6.1 to the study of the structure of the set of all $\lambda \in \mathbb{C} \backslash M_{F}$ for which the inequality in (6.26) is strict. More formally, we will show that if $K$ is a bounded Borel subset of positive logarithmic capacity then there exists a point $\lambda \in K \cap\left(\mathbb{C} \backslash M_{F}\right)$ such that equality holds true in (6.26). Furthermore, we will show that if $K$ is such a set and if $\nu$ is a finite Borel measure on $\mathbb{C}$ which is concentrated on $K$, i.e.,

$$
\nu(\mathbb{C} \backslash K)=0,
$$

and if the associated logarithmic potential $U^{(\nu)}$ (see (6.3)) is continuous in $\mathbb{C}$, then the identity

$$
\int_{\mathbb{C}}\left[v(\lambda)-v_{1-0}(\lambda)\right] \nu(d \lambda)=0
$$

is valid. Clearly, from (6.26) and (6.27) it will follow that $v(\lambda)=v_{1-0}(\lambda)$ for almost all $\lambda$ with respect to $\nu$. In particular, there exists a $\lambda \in K \cap\left(\mathbb{C} \backslash M_{F}\right)$ for which $v(\lambda)=v_{1-0}(\lambda)$ is satisfied. Now we are going to prove (6.27). According to (6.23) for $r \in[0,1)$ and $\lambda \in \mathbb{C}$ we have

$$
\int_{\mathbb{T}} \ln ^{+}\left|\operatorname{det}\left[F(r t)-\lambda I_{n}\right]\right| m(d t)-\int_{\mathbb{T}} \ln ^{-}\left|\operatorname{det}\left[F(r t)-\lambda I_{n}\right]\right| m(d t)=v_{r}(\lambda) .
$$


In view of Remark 6.5, the inequality

$$
\ln ^{+}\left|\operatorname{det}\left[F(r t)-\lambda I_{n}\right]\right| \leq n \cdot\left[\ln ^{+}\|F(r t)\|+\ln ^{+}|\lambda|+\ln 2\right]
$$

holds for $r \in[0,1), \lambda \in \mathbb{C}$ and $t \in \mathbb{T}$. For $\lambda \in \mathbb{C}$ and $r \in[0,1)$ the function $G_{\lambda, r}: \mathbb{T} \rightarrow$ $[0, \infty)$ is defined by

$$
G_{\lambda, r}(t):=\operatorname{det}\left[F(r t)-\lambda I_{n}\right] .
$$

Suppose that $\lambda \in \mathbb{C}$ is fixed. Then from (6.29) and (6.30) we infer that the family $\left(\ln ^{+}\left|G_{\lambda, r}\right|\right)_{r \in[0,1)}$ is uniformly $m$-integrable. Clearly, for almost all $t \in \mathbb{T}$ with respect to $m$ we have

$$
\lim _{r \rightarrow 1-0} \ln ^{+}\left|\operatorname{det}\left[F(r t)-\lambda I_{n}\right]\right|=\ln ^{+}\left|\operatorname{det}\left[\underline{F}(t)-\lambda I_{n}\right]\right| .
$$

Thus, using Vitali's convergence theorem again, we get

$$
\lim _{r \rightarrow 1-0} \int_{\mathbb{T}} \ln ^{+}\left|\operatorname{det}\left[F(r t)-\lambda I_{n}\right]\right| m(d t)=\int_{\mathbb{T}} \ln ^{+}\left|\operatorname{det}\left[\underline{F}(t)-\lambda I_{n}\right]\right| m(d t) .
$$

Taking into account (6.31) we obtain the formula

$$
\begin{aligned}
& \int_{\mathbb{T}} \ln ^{+}\left|\operatorname{det}\left[\underline{F}(t)-\lambda I_{n}\right]\right| m(d t)-\lim _{r \rightarrow 1-0} \int_{\mathbb{T}} \ln ^{-}\left|\operatorname{det}\left[F(r t)-\lambda I_{n}\right]\right| m(d t) \\
& =v_{1-0}(\lambda)
\end{aligned}
$$

by letting $r \rightarrow 1-0$ in (6.28), where the limit of the second term on the left hand side of (6.32) necessarily exists. From (6.25) and (6.32) it follows that

$$
\begin{aligned}
v(\lambda)-v_{1-0}(\lambda)= & \lim _{r \rightarrow 1-0} \int_{\mathbb{T}} \ln ^{-}\left|\operatorname{det}\left[F(r t)-\lambda I_{n}\right]\right| m(d t) \\
& -\int_{\mathbb{T}} \ln ^{-}\left|\operatorname{det}\left[\underline{F}(t)-\lambda I_{n}\right]\right| m(d t) .
\end{aligned}
$$

In general, the family $\left(\ln ^{-}\left|G_{\lambda, r}\right|\right)_{r \in[0,1)}$ is not uniformly $m$-integrable. For this reason, the right hand side in (6.33) is not necessarily zero. (However, according to Fatou's theorem this difference is nonnegative.) Nevertheless, it will turn out that after applying the following averaging procedure the right hand side of (6.33) vanishes. Suppose that $\nu$ is a finite nonnegative measure with compact support for which the associated logarithmic potential $U^{(\nu)}$ is continuous. We will prove that

$$
\int_{\mathbb{C}}\left(\lim _{r \rightarrow 1-0} \int_{\mathbb{T}} \ln ^{-}\left|\operatorname{det}\left[F(r t)-\lambda I_{n}\right]\right| m(d t)-\int_{\mathbb{T}} \ln ^{-}\left|\operatorname{det}\left[\underline{F}(t)-\lambda I_{n}\right]\right| m(d t)\right) \nu(d \lambda)=0 .
$$

Using Fubini's theorem and (6.11) we get

$$
\int_{\mathbb{C}}\left(\int_{\mathbb{T}} \ln ^{-}\left|\operatorname{det}\left[\underline{F}(t)-\lambda I_{n}\right]\right| m(d t)\right) \nu(d \lambda)=\int_{\mathbb{T}}\left(\int_{\mathbb{C}} \ln ^{-}\left|\operatorname{det}\left[\underline{F}(t)-\lambda I_{n}\right]\right| \nu(d \lambda)\right) m(d t)
$$




$$
=\int_{\mathbb{T}} \Phi_{-}^{(\nu)}(\underline{F}(t)) m(d t) .
$$

In view of (6.12) it follows that

$$
\int_{\mathbb{C}}\left(\int_{\mathbb{T}} \ln ^{-}\left|\operatorname{det}\left[\underline{F}(t)-\lambda I_{n}\right]\right| m(d t)\right) \nu(d \lambda)<\infty .
$$

Now we integrate identity (6.33) with respect to $\nu$ and use (6.36) to rewrite the integral of the difference as the difference of integrals. Then we rewrite the second term using (6.35) and apply Fatou's theorem to the first one. Finally, we use Fubini's theorem and (6.11) to rewrite the first term. This leads us to the following estimate

$$
\begin{aligned}
& \int_{\mathbb{C}}\left[v(\lambda)-v_{1-0}(\lambda)\right] \nu(d \lambda) \\
& =\int_{\mathbb{C}}\left[\lim _{r \rightarrow 1-0} \int_{\mathbb{T}} \ln ^{-}\left|\operatorname{det}\left[F(r t)-\lambda I_{n}\right]\right| m(d t)-\int_{\mathbb{T}} \ln ^{-}\left|\operatorname{det}\left[\underline{F}(t)-\lambda I_{n}\right]\right| m(d t)\right] \nu(d \lambda) \\
& =\int_{\mathbb{C}}\left[\lim _{r \rightarrow 1-0} \int_{\mathbb{T}} \ln ^{-}\left|\operatorname{det}\left[F(r t)-\lambda I_{n}\right]\right| m(d t)\right] \nu(d \lambda) \\
& -\int_{\mathbb{C}}\left[\int_{\mathbb{T}} \ln ^{-}\left|\operatorname{det}\left[\underline{F}(t)-\lambda I_{n}\right]\right| m(d t)\right] \nu(d \lambda) \\
& =\quad \int_{\mathbb{C}}\left[\lim _{r \rightarrow 1-0} \int_{\mathbb{T}} \ln ^{-}\left|\operatorname{det}\left[F(r t)-\lambda I_{n}\right]\right| m(d t)\right] \nu(d \lambda)-\int_{\mathbb{T}} \Phi_{-}^{(\nu)}(\underline{F}(t)) m(d t) \\
& \leq \quad \varliminf_{r \rightarrow 1-0} \int_{\mathbb{C}}\left[\int_{\mathbb{T}} \ln ^{-}\left|\operatorname{det}\left[F(r t)-\lambda I_{n}\right]\right| m(d t)\right] \nu(d \lambda)-\int_{\mathbb{T}} \Phi_{-}^{(\nu)}(\underline{F}(t)) m(d t) \\
& =\quad \varliminf_{r \rightarrow 1-0} \int_{\mathbb{T}}\left[\int_{\mathbb{C}} \ln ^{-}\left|\operatorname{det}\left[F(r t)-\lambda I_{n}\right]\right| \nu(d \lambda)\right] m(d t)-\int_{\mathbb{T}} \Phi_{-}^{(\nu)}(\underline{F}(t)) m(d t) \\
& =\varliminf_{r \rightarrow 1-0} \int_{\mathbb{T}} \Phi_{-}^{(\nu)}(F(r t)) m(d t)-\int_{\mathbb{T}} \Phi_{-}^{(\nu)}(\underline{F}(t)) m(d t) .
\end{aligned}
$$

According to Lemma 6.4 and our choice of $\nu$, the function $\Phi_{-}^{(\nu)}$ is continuous. Thus, for almost all $t \in \mathbb{T}$ with respect to $m$ we get

$$
\lim _{r \rightarrow 1-0} \Phi_{-}^{(\nu)}(F(r t))=\Phi_{-}^{(\nu)}(\underline{F}(t)) .
$$

Since the function $\Phi_{-}^{(\nu)}$ is also bounded (see Lemma 6.4), Lebesgue's theorem on dominated convergence and (6.38) guarantee

$$
\lim _{r \rightarrow 1-0} \int_{\mathbb{T}} \Phi_{-}^{(\nu)}(F(r t)) m(d t)=\int_{\mathbb{T}} \Phi_{-}^{(\nu)}(\underline{F}(t)) m(d t) .
$$


Thus, combining (6.37) and (6.39) we obtain (6.27).

As explained above this completes the proof.

COMMENTS ON THEOREM 6.1. These comments are intended to clarify the function-theoretic content of Theorem 6.1. Let $t \in \mathbb{T}$. Then the function $G: \mathbb{C} \rightarrow[-\infty, \infty)$ defined by

$$
G(\lambda):=\ln \left|\operatorname{det}\left[\underline{F}(t)-\lambda I_{n}\right]\right|
$$

is subharmonic. Let $r \in[0,1)$ and let the function $G_{r}: \mathbb{C} \rightarrow[-\infty, \infty)$ be defined by $G_{r}(\lambda):=G_{\lambda, r}(t)$, where $G_{\lambda, r}$ is given in (6.30). Then $G_{r}$ is subharmonic too. From standard theorems on integrating parametric families of subharmonic functions (see. e.g. Ronkin [Ron, Ch.I, §5] or Lelong and Gruman [LG, Appendix I, Proposition I.14]) it follows that the function $v$ defined in (6.25) is subharmonic and that for each $r \in[0,1)$ the function $v_{r}$ defined in (6.23) is also subharmonic. Since the family $\left(v_{r}\right)_{r \in[0,1)}$ increases monotonically with $r$, the function $v_{1-0}$ defined in (6.24) is the upper envelope of this family. The function $v_{1-0}$ is not necessarily subharmonic but its regularization $v_{1-0}^{*}: \mathbb{C} \rightarrow[-\infty, \infty)$ which is defined by

$$
v_{1-0}^{*}(\lambda):=\varlimsup_{\lambda^{\prime} \rightarrow \lambda} v_{1-0}\left(\lambda^{\prime}\right)
$$

turns out to be subharmonic (see Ronkin [Ron, Ch.I, §5], Lelong and Gruman [LG, Appendix I, Proposition I.25], Cartan [Car]). Clearly, for $\lambda \in \mathbb{C}$ the inequality

$$
v_{1-0}(\lambda) \leq v_{1-0}^{*}(\lambda)
$$

holds. According to an ingenious theorem of H. Cartan (see e.g. Ronkin [Ron, Ch.I, §5] or Cartan [Car]), the upper envelope of a family of subharmonic functions coincides with its regularization everywhere except for a set of logarithmic capacity zero. (For the exact formulation of Cartan's theorem and a proof we refer to Ronkin [Ron, Ch.I, §5]). In particular,

$$
\operatorname{cap}\left(\left\{\lambda: v_{1-0}^{*}(\lambda)>v_{1-0}(\lambda)\right\}\right)=0 .
$$

Since the function $v$ is upper semicontinuous (i.e., for $\lambda \in \mathbb{C}$, the inequality $v(\lambda) \geq$ $\varlimsup_{\lambda^{\prime} \rightarrow \lambda} v\left(\lambda^{\prime}\right)$ holds) the inequalities

$$
v_{1-0}(\lambda) \leq v_{1-0}^{*}(\lambda) \leq v(\lambda) .
$$

follows for $\lambda \in \mathbb{C}$ from (6.26). If we establish in some way that for all $\lambda$ belonging to some dense subset of $\mathbb{C}$ the equality $v_{1-0}(\lambda)=v(\lambda)$ holds true then in view of (6.43) we obtain

$$
v_{1-0}^{*} \equiv v .
$$

The identity $v_{1-0}(\lambda)=v(\lambda)$ for all $\lambda$ belonging to some dense subset of $\mathbb{C}$ clearly follows from the identity

$$
\int_{I}\left[v(\lambda)-v_{1-0}(\lambda)\right] \mu(d \lambda)=0,
$$

where $I$ is an arbitrary one dimensional interval of $\mathbb{C}$ and $\mu$ is one dimensional Lebesgue measure. The use of H. Cartan's theorem on upper envelopes of families of subharmonic 
functions for proving the smallness (in the sense of capacity) of exceptional sets has many traditions in the theory of functions of one or several complex variables. The application of the recently created complex potential theory, in particular the analogue of H. Cartan's theorem for the upper envelope of a family of plurisubharmonic functions (see Bedford and Taylor [BT , Section 7] and Sadullaev's survey paper [Sad]) enables one to derive results on families of matrix-valued functions of a more general type, namely on families which depend holomorphically on $p$ variables where $p \in \mathbb{N}$.

Finally, we turn our attention to the left version of our main result.

THEOREM 6.2. Let $F \in \mathfrak{N}_{n}^{+}(\mathbb{D})$. Assume that for $\lambda \in \mathbb{C} \backslash M_{F}$ the functions $\Theta_{\lambda, l} \in \mathfrak{I}_{n}(\mathbb{D})$ and $E_{\lambda, l} \in \mathfrak{E}_{n}(\mathbb{D})$ are factors in the multiplicative decomposition

$$
F_{\lambda}=E_{\lambda, l} \cdot \Theta_{\lambda, l} .
$$

Suppose that $K$ is a bounded Borel subset of $\mathbb{C}$ with positive logarithmic capacity. Then there exists a $\lambda \in K \cap\left(\mathbb{C} \backslash M_{F}\right)$ for which $\Theta_{\lambda, l}$ is a Blaschke-Potapov product.

PROOF. Use Theorem 6.1, Remark 3.2 and Remark 4.2.

COROLLARY 6.2. The set of all $\lambda \in \mathbb{C} \backslash M_{F}$ for which $\Theta_{\lambda, l}$ is a Blaschke-Potapov product is dense in $\mathbb{C}$.

For further matricial generalizations of the classical theorems of Frostman [Fr], Heins [Hei] and Rudin [Ru1] we refer the reader to the papers [Gi6] and [GiTa1] - [GiTa3].

\section{REFERENCES}

[Ar1] Arov, D.Z. : Darlington realization of matrix-valued functions (in Russian), Izv. Akad. Nauk SSSR, Ser. Mat. 37 (1973), 1299 - 1331, Engl. transl. in: Math. USSR Izvestija 7 (1973), 1295 - 1326, MR 50\#10287.

[Ar2] Arov, D.Z. : Functions of class $\Pi$ (in Russian), Zap. Nauc. Sem. LOMI 135 (1984), 5 - 30, Engl. transl. in : J. Soviet. Math. 31 (1985), 2645 - 2659, MR 85h:47041.

[Ar3] Arov, D.Z. : On regular and singular J-inner matrix-functions and related extrapolation problems (in Russian), Funkcional. Anal. i. Prilozhen. 22 (1988), no. 1, 57 - 59, Engl. transl. in: Functional Analysis and its Applic. 22 (1988), 46 - 48, MR 89d:47082.

[Ar4] Arov, D.Z. : $\gamma$-generating matrices, J-inner matrix-functions and related extrapolation problems I (in Russian), Teor. Funkcii, Funkcional. Anal. i. Prilozhen. 51 (1989), 61 - 67, Engl. transl. in: J. Soviet Math. 52 (1990), 3487 - 3491, MR 92i:30034a.

[Ar5] Arov, D.Z. : $\gamma$-generating matrices, J-inner matrix-functions and related extrapolation problems II (in Russian), Teor. Funkcii, Funkcional. Anal. i. Prilozhen. 21 (1989), 103 - 109, Engl. transl. in: J. Soviet Math. 52 (1990), 3421 - 3425, MR 92i:30034b. 
[Ar6] Arov, D.Z. : $\gamma$-generating matrices, J-inner matrix-functions and related extrapolation problems III (in Russian), Teor. Funkcii, Funkcional. Anal. i. Prilozhen. 53 (1990), 57 - 65, Engl. transl. in: J. Soviet Math. 52 (1992), 532 - 537, MR 92i:30034c.

[Ar7] Arov, D.Z. : Regular J-inner matrix-function and related continuation problems, in: Linear Operators in Function spaces (Eds.: Helson, H.; Sz.-Nagy, B.; Vasilescu, F.-H.), Operator Theory: Advances and Appl., vol. 43, Basel: Birkhäuser Verlag 1990, 63 - 87, MR 93b:47028.

[Ar8] Arov, D.Z. : The generalized bitangent Carathéodory - Nevanlinna - Pick problem and $\left(j, J_{0}\right)$-inner matrix-valued functions (in Russian), Izv. Rossiiskoi Akad. Nauk 57 (1993), 3 - 32, Engl. transl. in: Russian Acad. Sci. Izv. Math. 42 (1994), 1 - 26, MR 94j:47023.

[Ar9] Arov, D.Z.: Computation of the resolvent matrix for the generalized bitangential Schur - and Carathéodory - Nevanlinna - Pick interpolation problems in the strictly completely indeterminate case, Integral Equations and Operator Theory 22 (1995), 253 - 272

[AFK1] Arov, D.Z.; Fritzsche, B.; Kirstein, B. : On some completion problems for various subclasses of $j_{p q}$-inner functions, Zeitschrift für Analysis und ihre Anwend. 11 (1992), 489 - 508, MR 95d:30073.

[AFK2] Arov, D.Z.; Fritzsche, B.; Kirstein, B. : Completion problems for $j_{p q}$-inner functions, I, Integral Equations and Operator Theory 16 (1993), 155 - 185, MR 93k:47027.

[AFK3] Arov, D.Z.; Fritzsche, B.; Kirstein, B. : Completion problems for $j_{p q}$-inner functions, II, Integral Equations and Operator Theory 16 (1993), 453 - 495, MR 94h:47024.

[AFK4] Arov, D.Z.; Fritzsche, B.; Kirstein, B. : On block completion problems for various subclasses of $j_{p q}$-inner functions, in: Challenge of a Generalized Systems Theory (Eds.: Dewilde, P.; Kaashoek, A.; Verhaegen, M.A.), North Holland, Amsterdam 1993, pp. 179 - 194, MR 95h:30061.

[AFK5] Arov, D.Z.; Fritzsche, B.; Kirstein, B. : On block completion problems for $j_{q q^{-}}-J_{q^{-}}$ inner functions .I. The case of a given block column, Integral Equations and Operator Theory 18 (1994), 1 - 29, MR 95b:47016a.

[AFK6] Arov, D.Z.; Fritzsche, B.; Kirstein, B. : On block completion problems for $j_{q q^{-}}-J_{q^{-}}$ inner functions .II. The case of a given $q \times q$ block, Integral Equations and Operator Theory 18 (1994), 245 - 260, MR 95b:47016b.

[AFK7] Arov, D.Z.; Fritzsche, B.; Kirstein, B. : On some aspects of V.E. Katsnelson's investigations on interrelations between left and right Blaschke - Potapov products, in: Operator Theory and Boundary Eigenvalue problems (Eds.: Gohberg, I.; Langer H.), Operator Theory: Advances and Appl., vol. 80, Basel- Boston. Berlin: Birkhäuser Verlag 1995, pp. 21 - 41. 
[BT] Bedford, E.; Taylor, B.A. : A new capacity for plurisubharmonic functions, Acta Math. 149 (1982), 1 - 40, MR 84d:32024.

[Be] Beurling, A. : On two problems concerning linear transformation in Hilbert space, Acta Math. 81 (1949), 239 - 255, MR 10, p.381. Reprinted in:

Collected Works of Arne Beurling, Volume 1 Complex Analysis, Basel- Boston. Berlin: Birkhäuser Verlag 1989, pp. 147 - 163.

[Cam] Camera, G.A. : Nonlinear superposition on spaces of analytic functions, in: Harmonic Analysis and Operator Theory (Eds.: Marcantognini, S.A.M.; Mendoza, G.A.; Morán, M.D.; Octavio, A; Urbina, W.O.), Contemporary Mathematics, vol. 189 (1995), pp. 103 - 116, MR 95f:47093.

[Car] Cartan, H. : Théorie du potentiel newtonian : énergie, capacité, suites de potentiels, Bull. Soc. Math. France 73 (1945), 74 - 106, MR 7, p.447.

[CoLo] Collingwood, E.F., Lohwater, A.J. : The Theory of Cluster Sets, Cambridge: Univ. Press 1966, Russ. transl.: Moscow: Mir 1971, MR 38\#325.

[De] Devinatz, A. : The factorization of operator valued functions, Ann. Math. 73 (1961), 458 - 495, MR\#A3997.

[Doo1] Doob, J.L. : The boundary values of analytic functions, I, Trans. Amer. Math. Soc. 34 (1932), 153 - 170.

[Doo2] Doob, J.L. : The boundary values of analytic functions, II, Trans. Amer. Math. Soc. 35 (1933), 418 - 451.

[DFK] Dubovoj, V.K.; Fritzsche, B.; Kirstein, B. : Matricial Version of the Classical Schur Problem, Teubner Texte zur Mathematik 129, Stuttgart: B.G. Teubner 1992, MR 93e:47021.

[Dur] Duren, P.L. : Theory of $H^{p}$ Spaces, New York, London: Academic Press 1970, MR $42 \# 3552$.

[Fr] Frostman, O. : Potentiel d'équilibre et capacité des ensembles avec quelques applications à la théorie des fonctions, Medd. Lunds Univ. Math. Semin. 3 (1935), 1-118, MF 61, p.1262 (MF - Jahrbuch über die Fortschritte der Mathematik).

[G] Garnett, J.B. : Bounded analytic functions, New York, London: Academic Press 1981, MR 83g:30037.

[Gi1] Ginzburg, Yu.P. : The factorization of analytic matrix functions, Dokl. Akad. Nauk SSSR 159 (1964), 489 - 492; Engl. transl. in: Soviet Math. Dokl. 5 (1961), 1510 - 1514, MR 30\#3228.

[Gi2] Ginzburg, Yu.P. : Multiplicative representations of bounded analytic operator functions (in Russian), Dokl. Akad. Nauk SSSR 170 (1966), 23 - 26; Engl. transl. in: Soviet Math. Dokl. 7 (1966), 1125 - 1128, MR 34\#611. 
[Gi3] Ginzburg, Yu.P. : Multiplicative representations of operator functions of bounded form, Uspehi Mat. Nauk 22 (1967), no. 1, 163 - 165, MR 34\#6511.

[Gi4] Ginzburg, Yu.P. : Multiplicative representations and minorants of bounded analytic operator functions (in Russian), Funkcional. Anal. i Prilozhen. 1 (1967), no. 3, 9-23, MR 36\#4366 Engl. transl. in: Funkcional. Anal. and Appl. 1:3 (1967), 180-192.

[Gi5] Ginzburg, Yu.P. : Multiplicative representations of J-nonexpansive operator functions, Mat. Issled. 2 (1967), no. 2, 52 - 83; no. 3, 20 - 51; Engl. transl. in: Amer. Math. Soc. Transl. (Series 2) 96 (1970), 189 - 254, MR 38\#1551.

[Gi6] Ginzburg, Yu.P. : The almost invariant spectral properties of contractions and the multiplicative properties of analytic operator-functions (in Russian), Funkcional. Anal. i Prilozhen. 5 (1971), no. 3, 32 - 41, MR 44\#834. Engl. transl. in: Funkcional. Anal. and Appl. 5:3 (1971), 197 - 205.

[Gi7] Ginzburg, Yu.P. : On the reconstruction of a multiplicative integral from its modulus (in Russian), Teor. Funkcii, Funkcional. Anal. i Prilozhen. 41 (1984), MR 85k:47063.

[GiSh] Ginzburg, Yu.P.; Shevchuk, L.V. : On the Potapov theory of multiplicative representations, in: Matrix and Operator Valued Functions (Eds.: Gohberg, I.; Sakhnovich, L.A.), Operator Theory: Advances and Appl., vol. 72, Basel·Boston·Berlin: Birkhäuser Verlag 1994, pp. 28 - 47, MR 95k:47020.

[GiTa1] Ginzburg, Yu.P.; Taljusch, N.A. : A matricial analogue of a theorem of Heins and the typical special structure of contractions (in Russian), Funkcional. Anal. i Prilozhen. 7 (1973), no. 1, 66 - 67, MR 47\#4034 Engl. transl. in: Funkcional. Anal. and Appl. 7:1 (1973), 56 - 57.

[GiTa2] Ginzburg, Yu.P.; Taljusch, N.A. : On polynomial bundles of analytic matrix functions and families of contractive extensions of isometric operators (in Russian), Izvestiya Vuzov. Matematika 26 no. 4, ( 1982), 19 - 27, MR 84d:47009. Engl. transl. in: Soviet Mathematics (Izvestiya VUZ.Matematika) 26:4, 21 - 32.

[GiTa3] Ginzburg, Yu.P.; Taljusch, N.A. : Exceptional sets of analytic matrix functions, contractive and dissipative operators (in Russian), Izv. Vuzov 1984, no. 8, 9 14, Engl. transl. in: Soviet Mathematics (Izvestiya VUZ.Matematika) 28:8, 10 - 16. MR 87d:47067.

[Hal] Halmos, P. : Shifts on Hilbert spaces, J. reine und angew. Math. 208 (1961), 102 - 112, MR 27\#2868.

[Hei] Heins, M. : On the Lindelöf Principle, Ann. Math. 61 (1953), 440 - 473, MR 16, p.1011.

[Hel1] Helson, H. : Lectures on Invariant Subspaces, New York, London: Acad. Press 1964, MR 30\#1409. 
[Hel2] Helson, H. : Large analytic functions, in: Linear Operators in Function Spaces (Eds.: Helson, H.; Sz.-Nagy, B.; Vasilescu, F.-H.), Operator Theory: Advances and Appl., vol. 43, Basel- Boston· Berlin: Birkhäuser Verlag 1990, pp. 209 216, MR 92c:30038.

[Hel3] Helson, H. : Large analytic functions, II, in: Analysis and Partial Differential Equations: A Collection of Papers Dedicated to Mischa Cotlar (Ed.: Sadosky, C.), New York, Basel: Marcel Dekker 1990, pp. 217 - 220, MR 92c:30039.

[Hel4] Helson, H. : Large analytic functions, III, Colloq. Math., LX/LXI (1990), 221 223, MR 92c:30040.

[HL1] Helson, H.; Lowdenslager, D. : Prediction theory and Fourier series in several variables, I, Acta Math. 99 (1958), 165 - 202, MR 20\#4155.

[HL2] Helson, H.; Lowdenslager, D. : Prediction theory and Fourier series in several variables, II, Acta Math. 106 (1961), 175 - 213, MR 31\#562.

[HLP] Hardy, G.H.; Littlewood, J.E.; Pólya, G. : Inequalities, Cambridge: Cambridge Univ. Press 1934, MF 60, p.169-170.

[Hoff] Hoffman, K. : Banach spaces of analytic functions, Englewood Cliffs, N.J.: Prentice Hall 1962, MR 24\#A2844.

[Ka] Kato, T. : Perturbation theory for linear operators, Berlin, Heidelberg, New York: Springer Verlag 1966, MR 34\#3324.

[Kats1] Katsnelson, V.E. : A left Blaschke-Potapov product is not necessarily a right Blaschke-Potapov product (in Russian), Dokl. Akad. Nauk Ukrainian SSR, Series A 10 (1989), 15 - 17, MR 90k:47030.

[Kats2] Katsnelson, V.E. : Left and right Blaschke - Potapov products and Arov-singular matrix-valued functions, Integral Equations and Operator Theory 13 (1990), 836 - 848, MR 91f:47021.

[Kats3] Katsnelson, V.E. : Weighted spaces of pseudocontinuable functions and approximations by rational functions with prescribed poles, Zeitschrift für Analysis und ihre Anwend. 12 (1993), 27 - 67, MR 94m:30072.

[Kats4] Katsnelson, V.E. : Description of a class of functions which admit an approximation with preassigned poles I, in: Matrix and Operator Valued Functions (Eds.: Gohberg, I.; Sakhnovich, L.A.), Operator Theory: Advances and Appl., vol. 72, Basel-Boston-Berlin: Birkhäuser Verlag 1994, pp. 87 - 132, MR 96e:30095.

[Kh] Kheifets, A.J. : On regularization of $\gamma$-generating pairs, J. Functional Anal. 130 (1995), 310 - 333, MR 96b:47015. 
[Kol] Kolmogorov, A.N. : Stationary sequences in Hilbert space (in Russian), Bull. Math. Univ. Moscow 2 (1941), 1- 40, MR\#5101. Engl. transl. in: Selected Works of A.N. Kolmogorov. Vol. II: Probability Theory and Mathematical Statistics. (edited by A.N. Shiryaev), Dordrecht·Boston.London: Kluver Academic Publishers 1992, $228-271$.

[Koo] Koosis, P. : Introduction to $H^{p}$ Spaces, Cambridge: Cambridge University Press 1980, MR 81c:30062.

[Kr] Krein, M.G. : On a generalization of some investigations of G. Szegö, V.I. Smirnov and A.N. Kolmogorov (in Russian), Dokl. Akad. Nauk SSSR 46 (1945), 95 - 98, French transl. in: C.R. Acad. Sa. URSS (N.S.) 46 (1945), 91 - 94, $\mathrm{MR} \# 7156$.

[LVP1] de la Vallée Poussin, C. : Sur l'integrale de Lebesgue, Trans. Amer. Math. Soc. 16 (1915), 435 - 501, MF 45, p.441-442

[LVP2] de la Vallée Poussin, C. : Points irréguliers. Détermination des masses par les potentiels, Bull. de la class d. Sci. Acad. Belgique 24 (1938) 368 - 384, 672 689, MF 64, p.478, p.1162.

[LVP3] de la Vallée Poussin, C. : Le potentiel logarithmique, balayage et répresentation conforme, Paris: Gauthier-Villars 1949, Zbl. 37346.

[La] Lax, P. : Translation invariant subspaces, Acta Math. 101 (1959), 163 - 178, MR 21\#4359.

[LG] Lelong, P.; Gruman, L. : Entire functions of several complex variables, Berlin·Heidelberg·New York: Springer Verlag 1986, MR 90i:32002.

[MM] Marcus, M.; Minc, H. : A survey of matrix theory and matrix inequalities, Boston: Allyn and Bacon 1964, MR 29\#112.

[Ma1] Masani, P.R. : Cramér's theorem on monotone matrix-valued functions and the Wold decomposition, Probability and Statistics (U. Grenander, ed.) 175 - 189, New York: Wiley 1959, MR 23\#A2236.

[Ma2] Masani, P.R. : Shift invariant spaces and prediction theory, Acta Math. 107 (1962), 275 - 290, MR 25\#4344.

[Ma3] Masani, P.R. : Wiener's contributions to generalized harmonic analysis, prediction theory and filter theory, Bull. Amer. Math. Soc. 72 (1966), 73 - 125, MR $32 \# 4773$.

[Ma4] Masani, P.R. : Recent trends in multivariate prediction theory, in: Multivariate Analysis (Ed.: Krishnaiah, P.R.), New York, London: Academic Press 1966, pp. 351 - 382, MR 35\#5079.

[McC] McCarthy, J.E. : Topologies on the Smirnov class, J. Functional Analysis 104 (1992), 229 - 241, MR 93a:30041. 
[Mo1] Mochizuki, N. : Algebras of holomorphic functions between $H^{p}$ and $\mathfrak{N}_{*}$, Proc. Amer. Math. Soc. 105 (1989), 889 - 902, MR 90a:46137.

[Mo2] Mochizuki, N. : Nevanlinna and Smirnov classes on the upper half plane, Hokkaido Math. J. 20 (1991), 609 - 620, MR 93b:30031.

[Na] Nagumo, M. : Über die gleichmäßige Summierbarkeit und ihre Anwendung auf ein Variationsproblem, Japan J. Math. 6 (1929), 173 - 182, MF 55, p.156.

[Nic1] Nicolau, A. : The coefficients of Nevanlinna's parametrization are not in $H^{p}$, Proc. Amer. Math. Soc. 106 (1989), 115 - 117, MR 89k:30035.

[Nic2] Nicolau, A. : A characterization of the leading coefficient of Nevanlinna's parametrization, Illinois J. Math. 37 (1993), 284 - 301, MR 94c:30050.

[Nik1] Nikolskii, N.K. : Selected problems on weighted approximation and spectral analysis (in Russian), Trudy Math. Inst. Steklov 120 (1974), MR 57\#7133. Engl. transl. in: Proceedings of the Steklov Inst. of Math. Number 120 (1973), Providence, R.I.: AMS 1976.

[Nik2] Nikolskii, N.K. : Treatise on the Shift Operator, Berlin, Heidelberg, New York: Springer Verlag 1986, MR 87i:47042.

[No] Noshiro, K. : Cluster sets, Berlin·Göttingen·Heidelberg: Springer Verlag 1960, Russ. transl.: Moscow: Izd. inostrann. literatury 1963, MR 24\#A3295.

[Pot] Potapov, V.P. : The multiplicative structure of J-contractive matrix functions (in Russian), Engl. Transl. in: Amer. Math. Soc. Transl. (Series 2), 15 (1960), 131 - 243, MR 17, p.958-959.

[Pri] Privalov, I.I. : Boundary properties of analytic functions (in Russian), MR 13, p. 926, German transl.: Randeigenschaften analytischer Funktionen, Berlin: Deutscher Verlag der Wissenschaften 1956, MR 18, p.727.

[Rob] Roberts, J.W. : The component of the origin in the Nevanlinna class, Illinois J. Math. 19 (1975), 553 - 559, MR 52\#3554 / MR 18\#727.

[RoSt1] Roberts, J.W.; Stoll, M. : Prime and principal ideals in the algebra $\mathfrak{N}^{+}$, Arch. Math. 27 (1976), 387 - 393, Correction, ibid. 30 (1978), 672, MR 54\#10625.

[RoSt2] Roberts, J.W.; Stoll, M. : Composition operators on $F^{+}$, Studia Math. 57 (1976), 217 - 228, MR 55\#8773.

[Ron] Ronkin, L.I. : Introduction to the theory of entire functions of several variables (in Russian), Moscow: Nauka 1972, MR 47\#8896, English transl. in: Translation of Math. Monographs, vol. 44, AMS, Providence, R.I. 1974, MR 49\#10901.

[RoRo1] Rosenblum, M.; Rovnyak, J. : Hardy Classes and Operator Theory, Oxford Mathematical Monographs, Oxford: Clarendon Press 1985, MR 87e:47001. 
[RoRo2] Rosenblum, M.; Rovnyak, J. : Topics in Hardy Classes and Univalent Functions, Basel·Boston·Berlin: Birkhäuser Verlag 1994.

[Roz1] Rozanov, J.A. : Spectral properties of multivariate stationary processes and boundary properties of analytic matrices, Theory Probability Appl. (USSR), Engl. Transl. 5 (1960), 399 - 414, MR 24\#A2432.

[Roz2] Rozanov, J.A. : Stationary random processes, Moscow: Fizmatgiz 1963 (in Russian), Engl. translation: San Francisco: Holdon-Day 1967, MR 35\#4985.

[Ru1] Rudin, W. : A generalization of a theorem of Frostman, Math. Scand. 21 (1967), 136 - 143, MR 38\#3463.

[Ru2] Rudin, W. : Function Theory in Polydisc, New York, Amsterdam: Benjamin 1969, MR 41\#501.

[Ru3] Rudin, W. : Real and Complex Analysis, London: Mc Graw-Hill, London 1970, MR $32 \# 230$.

[Sad] Sadullaev, A. : Plurisubharmonic Functions, In: Several Complex Variables II: Encyclopaedia of Mathematical Sciences (Eds.: Khenkin, G.M.; Vitushkin, A.G.), Berlin, Heidelberg, New York: Springer Verlag 1994, pp. 59 - 106, MR 95e:32001.

[Sar1] Sarason, D. : Exposed points in $H^{1}$, in: The Gohberg Anniversary Collection, Volume II: Topics in Analysis and Operator Theory (Eds.: Dym, H.; Goldberg, S.; Kaashoek, M.A.; Lancaster, P.), Operator Theory: Advances and Apl., vol. 41, Basel·Boston· Berlin: Birkhäuser Verlag 1989, pp. 485 - 496, MR 91h:46043.

[Sar2] Sarason, D.: Sub-Hardy Hilbert Spaces in the Unit Disk, University of Arkansas Lecture Notes in the Math. Sciences, vol. 10, New York: Wiley 1995

[Sh] Shapiro, H.S. : Weakly invertible elements in certain function spaces and generators in $L^{1}$, Michigan Math. J., 11 (1964), 161 - 165, MR 29\#3620.

[ShSh] Shapiro, J.H.; Shields, A.L. : Unusual topological properties of the Nevanlinna class, Amer. J. Math. 97 (1976), 915 - 936, MR 52\#11053.

[Sm] Smirnov, V.I. : Sur les formules de Cauchy et de Green et quelques problèms qui s'y rattachent (in French), Izv. Akad. Nauk SSSR, Ser. Mat. 3 (1932), 338 - 372, Russian transl. in: Smirnov, V.I. : Selected Papers - Complex Analysis, Mathematical Theory of Diffraction (in Russian), Leningrad: Leningrad University Press 1988, pp. 82 - 111, MR 91g:01040.

[St1] Stoll, M. : A characterization of $F^{+} \cap \mathfrak{N}$, Proc. Amer. Math. Soc 57 (1976), 97 - 98, MR 53\#3315

[St2] Stoll, M. : Mean growth and Taylor coefficients of some topological algebras of analytic functions, Ann. Polon. Math. 35 (1977), 139 - 158, MR 57\#3858. 
[Sz1] Szegö, G. : Beiträge zur Theorie der Toeplitzschen Formen I, Math. Zeitschr. 6 (1920), 167 - 202, Reprinted in: Gabor Szegö - Collected Papers (Ed.: Askey, R.), vol. 1, Boston, Basel, Stuttgart: Birkhäuser Verlag 1982, pp. 237 - 272, MF 47, p.391

[Sz2] Szegö, G. : Beiträge zur Theorie der Toeplitzschen Formen II, Math. Zeitschr. 9 (1921), 167 - 190, Reprinted in: Gabor Szegö - Collected Papers (Ed.: Askey, R.), Volume 1, Boston, Basel, Stuttgart: Birkhäuser Verlag 1982, pp. 279 - 302,, MF 48, p.376-378

[Sz3] Szegö, G. : Über die Randwerte einer analytischen Funktion, Math. Ann. 84 (1921), 232 - 244, Reprinted in: Gabor Szegö - Collected Papers (Ed.: Askey, R.), Volume 1, Boston·Basel-Stuttgart: Birkhäuser Verlag 1982, pp. 404 - 416, MF 48, p.332

[SZNF] Sz.-Nagy B.; Foias, C. : Analyse Harmonique des Opérateurs de l'Espace de Hilbert, Budapest: Académiai Kiadó 1967, MR 37\#778, Engl. transl.: Harmonic Analysis of Operators on Hilbert Space, Budapest: Académiai Kiadó and Amsterdam, London: North-Holland Publishing Company 1970, MR $43 \# 947$.

[Vin] Vinogradov, S.A. : Properties of multipliers of Cauchy-Stieltjes integrals and some factorization problems for analytic functions (in Russian), in: Math. Programming and Related Questions (Proc. Seventh Winter School, Drogobych, 1974: Theory of Functions and Functional Analysis), Moscow: Central Econ.Math. Inst. Akad. Nauk SSSR 1976, pp. 5 - 39, Engl. transl. in: Amer. Soc. Transl. 115 (1980), 1 - 32, MR 58\#28518.

[Vit] Vitali, G. : Sull'integrazione per serie, Rend. Circ. Matem. Palermo 23 (1907), 137 - 155, MF 38, p.338.

[WM1] Wiener, N.; Masani, P. : The prediction theory of multivariate stochastic processes I. The regularity condition, Acta Math. 98 (1957), 111 - 150, Norbert Wiener - Collected Works (Ed.: Masani, P.R.), Volume III, Cambridge, MA; London: MIT Press 1981, pp. 164 - 203, MR 20\#4323.

[WM2] Wiener, N.; Masani, P. : The prediction theory of multivariate stochastic processes II. The linear predictor, Acta Math. 99 (1958), 93 - 137, Norbert Wiener - Collected Works (Ed.: Masani, P.R.), Volume III, Cambridge, MA; London: MIT Press 1981, 204 - 248, MR 20\#4325.

[Y1] Yanagihara, N. : On a class of functions and their integrals, Proc. London Math. Soc. (Third Series) 25 (1972), 550 - 576, MR 46\#3801.

[Y2] Yanagihara, N. : The second dual space for the space $\mathfrak{N}^{+}$, Proc. Japan Academy 49 (1973), 33 - 36, MR 49\#9600.

[Y3] Yanagihara, N. : Multipliers and linear functionals for the class $\mathfrak{N}^{+}$, Trans. Amer. Math. Soc. 180 (1973), 449 - 461, MR 49\#3147. 
[Y4] Yanagihara, N. : The containing Frechét space for the class $\mathfrak{N}^{+}$, Duke Math. J. 40 (1973), 93 - 103, MR 49\#9599.

[Y5] Yanagihara, N. : Bounded subsets of some spaces of holomorphic functions, Scientific Papers of the College of General Education, University of Tokyo 23 (1973), no. 1,19 - 28, MR 48\#2403.

[Y6] Yanagihara, N. : The class $\mathfrak{N}^{+}$of holomorphic functions and its containing Frechét space $\mathfrak{F}^{+}$, Boll. Un. Math. Ital. (Bologna) 8 (1973). 230 - 245, MR 48\#11520.

[Y7] Yanagihara, N. : Mean growth and Taylor coefficients of some classes of functions, Ann. Polon. Math. 30 (1974), 37 - 48, MR 49\#3148

[Y8] Yanagihara, N. : Interpolation theorems for the class $\mathfrak{N}^{+}$, Illinois J. Math. 18 (1974), 427 - 435, MR 50\#10271

[Y9] Yanagihara, N. : Generators and maximal ideals in some algebras of holomorphic functions, Tohoku Math. J. 27 (1975), 31 - 47, MR 52\#3555.

[Y10] Yanagihara, N. : Variational methods for functions of bounded characteristic, J. Math. Anal. Appl. 49 (1975), 561 - 574, MR 50\#13493.

[YK] Yanagihara, N.; Kawase, S. : On the characteristic of some classes of functions and their integrals, Proc. London Math. Soc. (Third Series) 25 (1972), 577 585, MR 47\#3681.

[YN] Yanagihara, N.; Nakamura, Y. : Composition operators on the class $\mathfrak{N}^{+}$, TRU Mathematics 14-2 (1978), 9 - 16, MR 80f:30024.

Victor Katsnelson

vDepartment of Theoretical Mathematics

The Weizmann Institute of Science

Rehovot, 76100

Israel

e-mail: katze@wisdom.weizmann.ac.il
Bernd Kirstein

Mathematisches Institut

Universität Leipzig

Leipzig, 04109

Bundesrepublik Deutschland

e-mail: heide@mathematik.uni-leipzig.de

AMS subject classification: 30D50, 46E10. 